\title{
Algebraically decaying modes and wave packet pseudo-modes in swept Hiemenz flow
}

\section{Journal Article}

Author(s):

Obrist, Dominik; Schmid, Peter J.

Publication date:

2010

Permanent link:

https://doi.org/10.3929/ethz-b-000017159

Rights / license:

In Copyright - Non-Commercial Use Permitted

Originally published in:

Journal of Fluid Mechanics 643, https://doi.org/10.1017/S0022112009992114 


\title{
Algebraically decaying modes and wave packet pseudo-modes in swept Hiemenz flow
}

\author{
DOMINIK OBRIST ${ }^{1} \dagger$ AND PETER J. SCHMID \\ ${ }^{1}$ Institute of Fluid Dynamics, ETH Zurich, 8092 Zürich, Switzerland \\ ${ }^{2}$ Laboratoire d'Hydrodynamique (LadHyX), CNRS-École Polytechnique, 91128 Palaiseau, France
}

(Received 27 March 2009; revised 3 September 2009; accepted 7 September 2009; first published online 23 December 2009)

The modal structure of the swept Hiemenz flow, a model for the flow near the attachment line of a swept wing, consists of eigenfunctions which exhibit (super-)exponential or algebraic decay as the wall-normal coordinate tends to infinity. The subset of algebraically decaying modes corresponds to parts of the spectrum which are characterized by a significant sensitivity to numerical discretization. Numerical evidence further suggests that a continuous spectrum covering a twodimensional range of the complex plane exists. We investigate the family of uniform swept Hiemenz modes using eigenvalue computations, numerical simulations and the concept of wave packet pseudo-modes. Three distinct branches of the family of algebraically decaying eigenmodes are identified. They can be superimposed to produce wavefronts propagating towards or away from the boundary layer and standing or travelling wave packets in the free stream. Their role in the exchange of information between the free stream and the attachment-line boundary layer for the swept Hiemenz flow is discussed. The concept of wave packet pseudo-modes has been critical in the analysis of this problem and is expected to lead to further insights into other shear flows in semi- or bi-infinite domains.

Key words: instability, stability

\section{Introduction}

The swept Hiemenz flow has long been acknowledged as a model for the flow near the attachment line of swept wings. As such it has received a great deal of attention from theoreticians, numericists and experimentalists alike, since findings about its stability characteristics have a direct impact on aeronautical and nautical applications as well as vehicle engineering. Despite remarkable progress, many questions about the stability behaviour (and spectrum) of this flow remain open. In particular, the role of the continuous spectrum, the influence of free-stream perturbations and the significance of algebraically decaying modes are still unclear and debated (see, e.g., the discussion in $\S 3$ of Hall, Malik \& Poll 1984). Without any sweep, i.e. for a plane Hiemenz flow, the existence of a continuous spectrum with modes which decay algebraically in the upstream direction has been acknowledged already by Hämmerlin (1955). However, Kestin \& Wood (1970) have argued that Hämmerlin's results are due only to 'an excessive idealization' of the problem. Wilson \& Gladwell (1978) dismissed algebraic modes as irrelevant for actual attachment-line boundary

$\dagger$ Email address for correspondence: obrist@ifd.mavt.ethz.ch 
layers mainly because the vorticity in the base flow decays exponentially in the free stream and instabilities are confined to the boundary layer. Therefore, they imposed boundary conditions which enforce exponential decay. This view was adopted by several other authors (Lyell \& Huerre 1985; Brattkus \& Davis 1991), which excluded algebraically decaying modes in order to obtain a discrete eigenvalue spectrum. In contrast, Dhanak \& Stuart (1995) argued that modes with algebraic decay or growth in the upstream direction are physically relevant for plane stagnation-point flow because they 'match with the external flow'. For the swept Hiemenz flow, most authors (Hall et al. 1984; Hall \& Malik 1986; Lin \& Malik 1996; Spalart 1988; Theofilis 1998; Obrist 2000) suppressed algebraically decaying modes either explicitly by imposing the exponential decay through a boundary condition or implicitly by truncating the computational domain in the upstream direction and imposing zero boundary conditions. Obrist \& Schmid (2003a) presented eigenvalue spectra which contain modes with exponential and algebraic decay. Further, they demonstrated that algebraically decaying modes are relevant for transient growth phenomena in the swept Hiemenz flow (Obrist \& Schmid 2003b). Although their findings suggested that the algebraically decaying modes form a continuous spectrum for the swept Hiemenz flow as well, the results were inconclusive.

This paper contributes to the discussion about algebraically decaying modes in the swept Hiemenz flow by applying the novel technique of wave packet pseudomodes (Trefethen 2005; Trefethen \& Embree 2005) to a class of swept Hiemenz modes known as the 'uniform modes'. This technique is a microlocal approach to stability theory and allows the treatment of a variety of complex fluid stability problems. In our specific case, it provides insight into the structure, nature and role of modal solutions that decay algebraically in the free stream. Wave packet pseudo-modes have been introduced in an attempt to compute approximations to the local dispersion relation of ordinary differential equations with variable coefficients. The technique has yielded results that provide insight into the dynamic behaviour of this type of differential equations, and we expect the same to hold for the differential equation governing the evolution of uniform modes in the swept Hiemenz flow.

We will introduce the governing eigenvalue problem in $\S 2$ and will demonstrate in $\S 3$ that it is not possible to study the algebraically decaying eigenmodes simply by solving for the eigenvalues of the discretized operator. Therefore, for determining such eigenfunctions we will outline a robust method which is based on the singularvalue decomposition of the discretized operator and on asymptotic results for the algebraically decaying modes. We will use this method to study a variety of algebraically decaying modes and will make the observation that all these modes consist of one or two wave packets. In $\S 4$, we will introduce the theory of wave packet pseudo-modes. It will be shown in $\S 4.2$ that the wave packet pseudo-modes of the swept Hiemenz flow approximate the algebraically decaying modes. Therefore, we can use the results of the theory of wave packet pseudo-modes to describe the structure of the spectrum of the algebraically decaying modes. Section 5 will use the results from the previous sections to discuss the role of the algebraically decaying modes in the dynamics of the swept Hiemenz flow. We will find that these modes are responsible for the communication between the free stream and the boundary layer. In $\S 5.3$, the theory of wave packet pseudo-modes will help us to make predictions about the dynamics of free-stream perturbations travelling towards the boundary layer. Section 6 concludes the paper. 


\section{Uniform modes in swept Hiemenz flow}

The temporal eigenvalue problem for the swept Hiemenz flow (Hall et al. 1984; Obrist \& Schmid 2003a) yields a number of eigensolutions $(u, v, w)$ that can be classified by their polynomial order $p$ in the chordwise direction, $(u, v, w) \sim\left(x^{p}, x^{p-1}, x^{p-1}\right)$, and by their decay in the free stream. (For an illustration of the base flow, the coordinate directions and the velocity components we refer the reader to figure 1 of Obrist \& Schmid 2003a.) While most relevant modes, including the most unstable mode named after Görtler (1941) and Hämmerlin (1955), decay either exponentially or even super-exponentially fast $\left(\sim \exp \left(-y^{2} / 2\right)\right)$ in the free stream, there exists a large number of eigensolutions with only algebraic decay. For eigensolutions following the Görtler-Hämmerlin ansatz ( $p=1$, i.e. $u$ is linear and $v$ and $w$ are constant in $x$ ) the existence of algebraically decaying modes was first reported by Hall et al. (1984). Obrist \& Schmid (2003a) showed that they do exist for all orders $p \geqslant 0$. At present, however, the physical role of the algebraically decaying modes remains unclear. Arguments for their dismissal as artefacts of the unbounded nature of the base flow (Wilson \& Gladwell 1978) coexist with arguments for their relevance in fully describing the flow near the attachment line (Dhanak \& Stuart 1995). Despite various efforts, it has not been possible so far to determine the shape of the eigenvalue spectrum associated with the algebraically decaying modes. It was conjectured (Obrist \& Schmid 2003a) that the eigenvalues fill the whole half-plane below an upper bound for the imaginary value.

We will address the question of the spectrum for algebraically decaying modes by considering the uniform swept Hiemenz modes $(p=0)$. These modes are technically the simplest set of eigensolutions for the swept Hiemenz flow but possess nevertheless all relevant features of algebraically decaying eigensolutions. Uniform modes in the swept Hiemenz flow have the general form

$$
(u, v, w)=(\hat{u}(y), 0,0) \mathrm{e}^{\mathrm{i}(\gamma z-\lambda t)} .
$$

Together with the basic flow field $(x U(y) / R, V(y) / R, W(y))$, this ansatz yields the following eigenvalue problem for the uniform modes:

$$
\left[\mathscr{D}^{2}-V \mathscr{D}+V^{\prime}-\gamma^{2}-\mathrm{i} \gamma R W\right] \hat{u}=-\mathrm{i} R \lambda \hat{u},
$$

where $\mathscr{D}=\mathrm{d} / \mathrm{d} y, V^{\prime}=\mathrm{d} V / \mathrm{d} y$ and $R$ is the Reynolds number. The boundary conditions are $\hat{u}(0)=\hat{u}(y \rightarrow \infty)=0$. In contrast to higher-order modes, the linear equation (2.2) is exact in the domain of the Hiemenz boundary layer and its associated stagnation point flow. It can be obtained directly from the Navier-Stokes equations without linearization.

\section{Algebraically decaying uniform modes}

It is known that the eigenvalue problem (2.2) permits two fundamental types of solutions: (a) modes with algebraic decay, $\hat{u} \sim y^{-v-1}$, and $(b)$ modes with superexponential decay, $\hat{u} \sim y^{v} \exp \left(-y^{2} / 2\right)$, as $y \rightarrow \infty$ with $v$ defined as

$$
v=-2-\gamma^{2}-\mathrm{i} R(\gamma-\lambda) \text {. }
$$

Obrist \& Schmid (2003a) presented numerical and analytical evidence that the superexponentially decaying modes correspond to the discrete eigenvalues and to the infinitely many eigenvalues located along a continuous line spectrum with $\lambda_{r}=\gamma$ and 
(a)
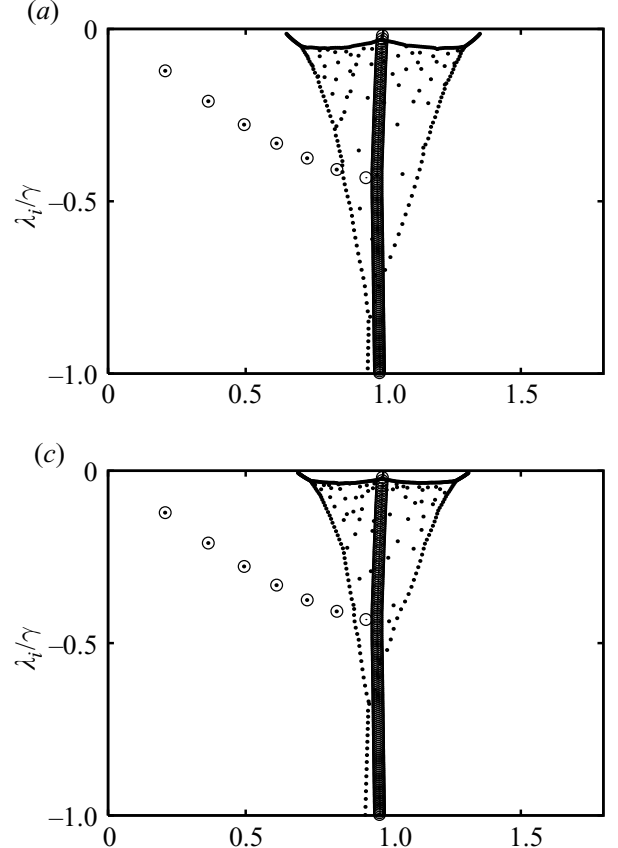

(e)

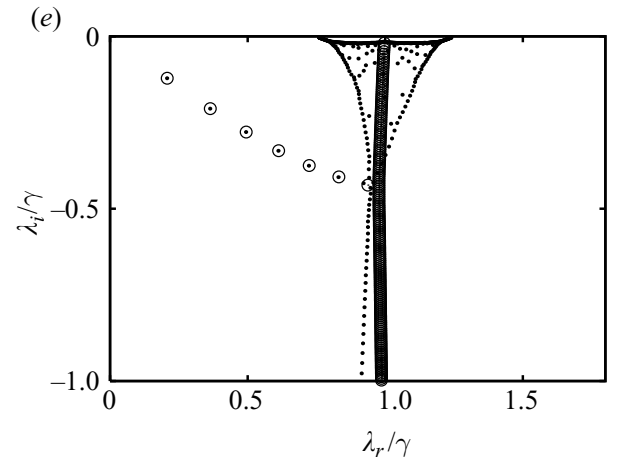

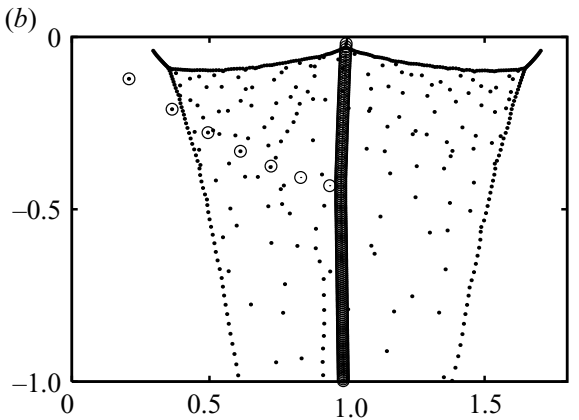

(d)
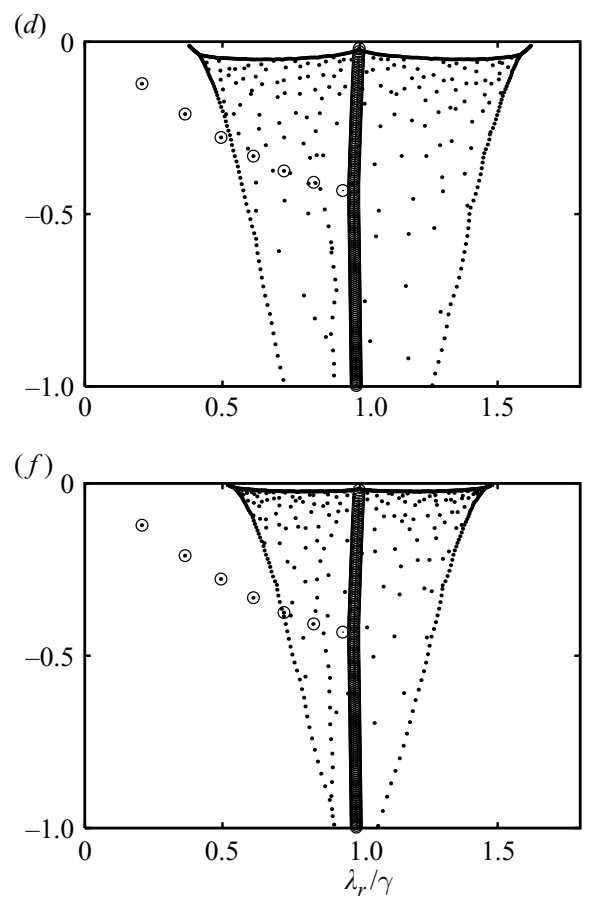

FIGURE 1. Numerically determined eigenvalues $(\cdot)$ for the uniform problem $(R=1000, \gamma=0.3)$ on a logarithmically stretched grid with $N=500$ discretization points changing domain size $L:$ : a $L=100$, (c) $L=200$ and (e) $L=1000$. The same computations on a logarithmically stretched grid with $N=1000$ discretization points, again with $(b) L=100,(d) L=200$ and ( $f) L=1000$. The circles (o) indicate the modes with super-exponential decay (including the eigenvalues along the continuous line spectrum $\left.\lambda_{r} / \gamma=1\right)$.

$\lambda_{i}<0$, where $\lambda=\lambda_{r}+\mathrm{i} \lambda_{i}$. Similar relations for the algebraically decaying modes could not be obtained; only an upper bound for the eigenvalues of the form

$$
\lambda_{i}<-\frac{1+\gamma^{2}}{R}<0
$$

could be given. Numerical experiments, however, suggest that these eigenvalues form a continuous spectrum which covers a substantial 'area' in the stable complex halfplane. The exact shape and structure of the spectrum, however, remain unclear.

We document this fact by plotting numerically evaluated eigenvalues of (2.2) for $R=1000$ and $\gamma=0.3$ with different discretizations (figure 1 ). We have used secondorder central finite differences to discretize the equation on a grid with exponential 

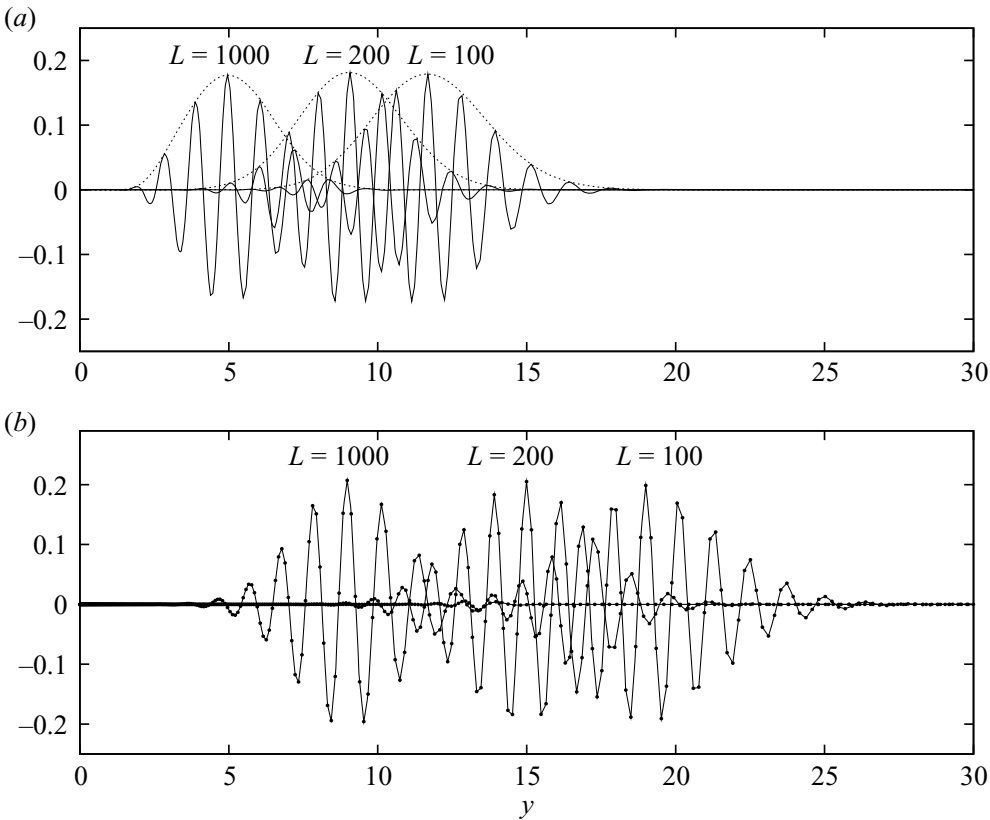

FiguRE 2. Examples of numerically evaluated eigenfunctions on grids with $N=500$ and $L=100,200$ and $1000(R=1000, \gamma=0.3):(a)$ modes located inside the kite-shaped regions of figure $1(L=100$ with $\lambda / \gamma=1.198-0.113 \mathrm{i} ; L=200$ with $\lambda / \gamma=1.154-0.117 \mathrm{i} ; L=1000$ with $\lambda / \gamma=1.079-0.113 \mathrm{i}) ;(b)$ marginally resolved eigenfunctions corresponding to eigenvalues which are located at the edge of the kite-shaped regions $(L=100$ with $\lambda / \gamma=1.294-0.104 \mathrm{i}$; $L=200$ with $\lambda / \gamma=1.234-0.106 \mathrm{i} ; L=1000$ with $\lambda / \gamma=1.137-0.100 \mathrm{i})$. Because all eigenvalues have almost the same imaginary value $\left(\lambda_{i} / \gamma \approx-0.1\right)$ the algebraic decay $y^{-\mu}$ is nearly the same for all shown eigenfunctions.

mapping, $y_{j}=\exp [j /(N-1) \cdot \log (L+1)]-1, j=0, \ldots, N-1$. Figure 1 also displays the eigenvalues which correspond to super-exponentially decaying modes. These modes were obtained from a modified eigenvalue problem which excludes all algebraically decaying modes (for details, see Obrist 2000; Obrist \& Schmid 2003a).

The eigenvalues of the super-exponentially decaying modes (seven discrete eigenvalues and a continuous line spectrum) are insensitive to the discretization. The eigenvalues of the algebraically decaying modes, however, depend strongly on the discretization. The kite-shaped region with its apex close to $\lambda=\gamma$ becomes larger as we increase the number of grid points - commonly taken as a sign of spurious results. Nevertheless, these eigenvalues are not numerical artefacts. Inspection of the associated eigenfunctions (figure $2 a$ ) confirms that they all correspond to algebraically decaying solutions of the uniform eigenvalue problem (2.2). The displayed eigenvalues appear to belong to a continuous spectrum which spans a large region in the complex plane. Based on our discretization of the eigenvalue problem (2.2) only a small portion of this continuous spectrum can be determined. Our discretization using $N$ grid points leads to at most $N$ eigenvalues sampled from the continuous spectrum; the exact location of the converged eigenvalues from this part of the spectrum depends on the details of our discretization. Although the eigenvalue solver does not converge for eigenvalues outside of the kite-shaped region, there may exist eigensolutions of the continuous $(N \rightarrow \infty)$ eigenvalue problem (2.2). 
In the free stream, the algebraically decaying eigenfunctions can be regarded as harmonic waves with exponential decay on the logarithmic coordinate $\eta=\ln y$,

$$
\hat{u} \sim y^{-\nu-1}=\exp \left[\left(1+\gamma^{2}+R \lambda_{i}\right) \eta+\mathrm{i} R\left(\gamma-\lambda_{r}\right) \eta\right] .
$$

In that sense, we can interpret the real part of the exponential coefficient as a decay rate $\mu$ and the imaginary part as a wall-normal wavenumber $\beta_{\eta}$ (the subscript $\eta$ indicates that this is a wavenumber in logarithmic coordinates),

$$
\begin{aligned}
& \mu=-\left(1+\gamma^{2}+R \lambda_{i}\right), \\
& \beta_{\eta}=R\left(\gamma-\lambda_{r}\right) .
\end{aligned}
$$

With these definitions we can state that eigenvalues outside the kite-shaped regions of figure 1 correspond to solutions with (possibly very slow) decay rates $\mu$ and (possibly very high) wavenumbers $\beta_{\eta}$ which are not resolved by the chosen discretization. The edges of the kite-shaped regions consist of eigenfunctions which are barely resolved on the chosen grid (figure $2 b$ ).

The observed sensitivity of the exact eigenvalues to small perturbations, in our case introduced by the finite-difference approximation of the eigenvalue problem, is not of primary concern but is rather an indication of an operator quantity which gives important insight into the behaviour of perturbations in the free stream and their interaction with the boundary layer. Instead of an analysis of discretization errors on the location and convergence of the algebraically decaying eigensolutions, we thus take a different approach by asking whether a chosen value of $\lambda \in \mathbb{C}$ and a certain function $\hat{u}$ satisfy, to a given degree, the eigenvalue problem (2.2).

To this end, we let ourselves be guided by (3.3) and (3.4) to select the asymptotic behaviour of $\hat{u}$ for large $y$. The same relations will also tell us how to best discretize the eigenvalue problem (2.2): the logarithmic coordinate $\eta$ in (3.3) suggests an exponential mapping of the grid points, and the decay rate $\mu$ provides a value for the upper limit $L$ of the computational domain. In practice, we set $L$ to allow a decay of the eigenfunction amplitude by at least 16 orders of magnitude. Finally, the wavenumber $\beta_{\eta}$ yields a bound on the number of grid points per (logarithmic) length unit to properly resolve the function $\hat{u}$. With all this information at hand, we discretize the continuous eigenvalue problem (2.2) and obtain

$$
L w=\lambda w,
$$

where $\boldsymbol{L}$ and $\boldsymbol{w}$ are the discrete forms of the differential operator in (2.2) and of $\hat{u}$, respectively. If $\lambda$ is an eigenvalue of (2.2) the matrix $\boldsymbol{L}-\lambda \boldsymbol{I}$ is singular. As we determine the spectrum of $\boldsymbol{L}$, we should therefore look for zero singular values $\sigma$ in the singular-value decomposition of our discretized operator $\boldsymbol{L}-\lambda \boldsymbol{I}$,

$$
\boldsymbol{U} \Sigma \boldsymbol{V}^{H}=\boldsymbol{F}(\boldsymbol{L}-\lambda \boldsymbol{I}) \boldsymbol{F}^{-1},
$$

where the matrix $\boldsymbol{F}$ has been introduced to account for numerical integration weights which are chosen such that the vector norm $\|\boldsymbol{q}\|_{E} \equiv\|\boldsymbol{F} \boldsymbol{q}\|_{2}$ represents the discretized equivalent of the continuous norm

$$
\|q\|_{\mathscr{\delta}}=\int_{0}^{\infty} q(y)^{*} q(y) \mathrm{d} y .
$$

In theory, the matrix $\boldsymbol{F}$ could be omitted, since the smallest singular value will be exactly zero (independent of the choice of $\boldsymbol{F}$ ) if $\lambda$ is an eigenvalue. In practice, however, the smallest singular value will never be exactly zero (owing to finite-precision 

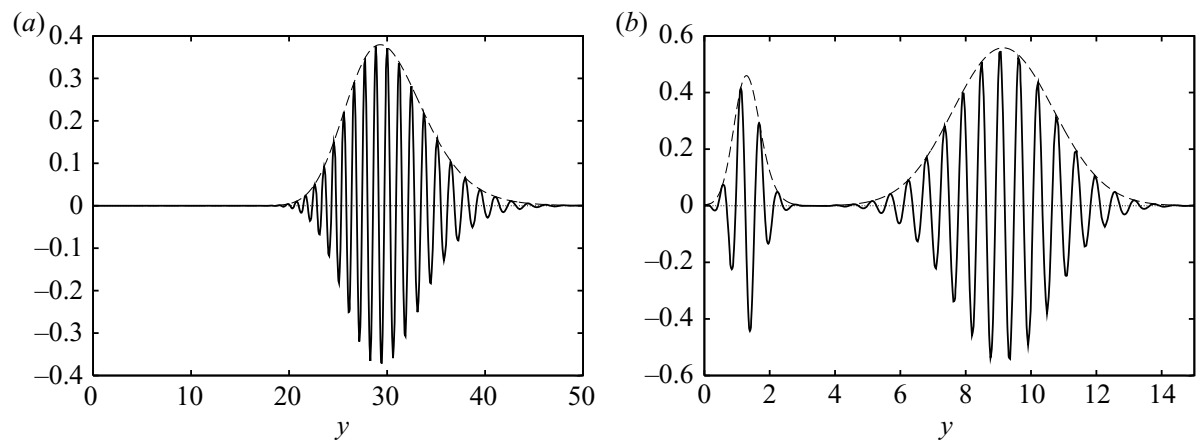

FIGURE 3. Algebraically decaying eigenfunction for $(a) \lambda=(1.5-0.1 \mathrm{i}) \gamma$ and $(b)$ $\lambda=(0.7-0.4 \mathrm{i}) \gamma$ with $\gamma=0.3$ and $R=1000$ ( - , real part; --- , absolute value).

arithmetic and discretization errors), and $\boldsymbol{F}$ provides a domain-independent metric to measure the closeness-to-zero of the singular value and thereby the degree to which $\{\lambda, \boldsymbol{w}\}$ satisfies the discretized eigenvalue problem.

From the singular-value decomposition (3.6) we find that

$$
(\boldsymbol{L}-\lambda \boldsymbol{I})\left(\boldsymbol{F}^{-1} \boldsymbol{v}_{N}\right)=\sigma_{N} \boldsymbol{F}^{-1} \boldsymbol{u}_{N}
$$

where $\sigma_{N}$ denotes the smallest singular value with its corresponding singular vectors $\boldsymbol{u}_{N}$ and $\boldsymbol{v}_{N}$. This shows that for a given choice of $\lambda$ the expression $\boldsymbol{F}^{-1} \boldsymbol{v}_{N}$ constitutes the optimal approximation to an eigenvector of $\boldsymbol{L}$ (optimal with respect to the norm $\left.\|\cdot\|_{E}\right)$. The norm of the right-hand side $\sigma_{N} \boldsymbol{F}^{-1} \boldsymbol{u}_{N}$ indicates the amount by which $\boldsymbol{F}^{-1} \boldsymbol{v}_{N}$ deviates from an actual eigensolution of $\boldsymbol{L}$. By definition of the singular-value decomposition, the norm of the right-hand side is given by $\sigma_{N}$,

$$
\left\|\sigma_{N} \boldsymbol{F}^{-1} \boldsymbol{u}_{N}\right\|_{E}=\sigma_{N}\left\|\boldsymbol{F} \boldsymbol{F}^{-1} \boldsymbol{u}_{N}\right\|_{2}=\sigma_{N}\left\|\boldsymbol{u}_{N}\right\|_{2}=\sigma_{N} .
$$

Therefore, we can consider $\left\{\lambda, \boldsymbol{F}^{-1} \boldsymbol{v}_{N}\right\}$ a genuine eigensolution if the associated smallest singular value $\sigma_{N}$ is zero (or at least equivalent to zero in finite-precision arithmetic).

Figures 3 and 4 show some eigenfunctions $\boldsymbol{F}^{-1} \boldsymbol{v}_{N}$ determined by the procedure outlined above. We call them eigenfunctions because for all chosen values of $\lambda$ (for which (3.2) is satisfied) the associated singular values $\sigma_{N}$ were smaller than machine precision. These solutions are further confirmed by direct numerical simulations of (2.2) with $\boldsymbol{F}^{-1} \boldsymbol{v}_{N}$ as the initial condition. For these simulations, we use a third-order Runge-Kutta scheme for the integration in time and a second-order finite-difference scheme for the discretization in space. These tests demonstrate that $\boldsymbol{F}^{-1} \boldsymbol{v}_{N}$ are genuine eigenmodes; i.e. they maintain their spatial shape while their amplitude decreases in time according to $\exp \left(\lambda_{i} t\right)$. From a physical point of view, this finding appears quite surprising, since some of these modes are located far from the wall, where we experience a strong wall-normal base flow. Intuitively, we would have expected that the base flow advects all disturbances towards the wall ( $\$ 5.3$ will continue along this thought).

Not surprisingly, the shapes of all these eigenfunctions comply with the asymptotic result (3.3) as demonstrated in figure $4(b)$. Even for $\lambda$ chosen to coincide with the location of super-exponential eigensolutions (indicated by circles in figure 1) we can find algebraically decaying eigensolutions.

Further, we notice that all these eigenfunctions have the shape of wave packets. The peaks of the wave packets appear to move farther away from the wall as $\left|\lambda_{r}-\gamma\right|$ 

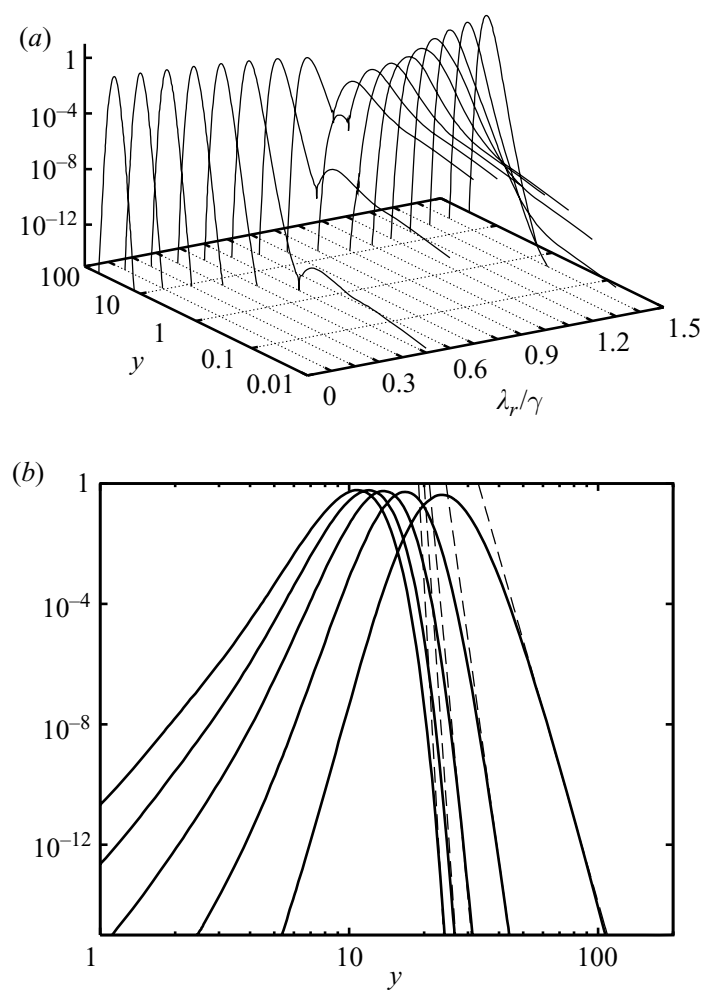

FIGURE 4. Absolute values of the algebraically decaying eigenfunctions for $(a) \lambda=\lambda_{r}-0.1 \mathrm{i} \gamma$ and $(b) \lambda=1.4 \gamma+\mathrm{i} \lambda_{i}$ where $\lambda_{i} / \gamma$ takes the values $-0.5,-0.4,-0.3,-0.2,-0.1$ (from left to right). The broken lines indicate the algebraic decay $y^{-\mu}(\gamma=0.3, R=1000)$.

increases and/or as $\lambda_{i}$ increases (figure 4). Some eigenfunctions feature two wave packets: one in the free stream and one inside the boundary layer (see figures $3 b$ and $4 a$ ). Moreover we find that in general the dominant wavenumber of these wave packets is not equal to $\beta_{\eta}$. This wavenumber is only approached in the limit as $y$ tends to infinity, which is also where we find the predicted algebraic decay, $\sim y^{-\mu}$ (broken lines in figure $4 b$ ).

The results of this section present strong numerical evidence that algebraically decaying modes exist in a large region of the complex plane bounded by (3.2). This region also includes modes with $\lambda_{r}<0$ and $\lambda_{r}>\gamma$. Our numerical procedure for computing these modes revealed no bounds on $\lambda$ other than (3.2). The following section will introduce a framework for analysing the spectral properties of algebraically decaying modes and offer an explanation for the structure of the spectrum as well as the particular shapes of the eigenfunctions.

\section{Wave packet pseudo-modes}

The assertion of a continuous spectrum based on algebraically decaying modes, which cannot be sufficiently resolved by numerical techniques, can be further substantiated by using wave packet pseudo-modes, a concept introduced by Trefethen (2005) (see also Trefethen \& Embree 2005) to analyse the spectral properties of variable-coefficient differential operators. Wave packet pseudo-modes are local solutions that 'exactly' satisfy the dispersion relation of the differential operator but only 'approximately' satisfy the boundary conditions. They are closely linked to 
Wentzel-Kramers-Brillouin-Jeffreys (WKBJ) approximations to the eigenmodes of the differential equation. The mathematical framework developed in this section will provide insight into the shape of the eigenfunctions and the location of the continuous spectrum in the complex plane. It will further build the basis for a more physical interpretation of the role of algebraically decaying eigenfunctions.

\subsection{Symbol and twist condition}

Wave packet pseudo-modes $\hat{u}$ are localized about the coordinate $y_{*}$ and the wavenumber $\beta_{*} / \epsilon$. They are asymptotically good solutions to the eigenvalue problem $(\mathscr{L}-\lambda) \hat{u}=0$ in the sense that they satisfy

$$
\frac{\|(\mathscr{L}-\lambda) \hat{u}\|}{\|\hat{u}\|} \leqslant M^{-1 / \epsilon} \quad \text { for } M>1
$$

and

$$
\frac{\left|\hat{u}\left(y ; y_{*}, \beta_{*}\right)\right|}{\max \left|\hat{u}\left(y ; y_{*}, \beta_{*}\right)\right|} \leqslant C_{1} \mathrm{e}^{-C_{2}\left(y-y_{*}\right)^{2} / \epsilon} \quad \text { for } C_{1}>0,
$$

for a sufficiently small asymptotic parameter $\epsilon$. If $\hat{u}$ behaves around $y_{*}$ like $\mathrm{e}^{\mathrm{i} \beta_{v} y / \epsilon}$, it satisfies locally an algebraic dispersion relation that depends on $y_{*}$ as well as $\beta_{*}$. This dispersion relation is given by the 'symbol' $f\left(y_{*}, \beta_{*}\right)$. The symbol can be interpreted as an asymptotic approximation to a pseudo-eigenvalue associated with a corresponding pseudo-mode in wave packet form. Of course not all values of $y_{*}$ and $\beta_{*}$ produce a value $\lambda=f\left(y_{*}, \beta_{*}\right)$ that approximates an eigenvalue of the original system. We are specifically interested in solutions that decay as $\left|y-y_{*}\right|$ increases; in other words, the coefficient $C_{2}$ in $(4.1 b)$ has to be positive. This requirement leads to an inequality, known as the 'twist condition', which constrains the range of $y_{*}$ and $\beta_{*}$ that allow wave packet pseudo-modes. It can be expressed in general form as

$$
\operatorname{Im}\left\{\frac{\partial f}{\partial y_{*}} / \frac{\partial f}{\partial \beta_{*}}\right\}<0 .
$$

The dispersion relation $\lambda=f\left(y_{*}, \beta_{*}\right)$ together with the twist condition (4.2) thus approximates eigenvalues in the complex plane whose associated eigenfunctions have the form of wave packets and satisfy the correct boundary conditions up to an error that decreases exponentially as $\epsilon$ tends to zero (for more details, see Trefethen \& Embree 2005). The mapping of $\left(y_{*}, \beta_{*}\right)$ to $\lambda$ under observation of the twist condition will result in distinct branches of the dispersion relation and, subsequently, in the interpretation of the continuous spectrum in terms of propagating and standing waves.

For our specific case of the uniform swept Hiemenz problem (2.2) the symbol can be derived as

$$
f\left(y_{*}, \beta_{*}\right)=-\mathrm{i} \beta_{*}^{2}+\epsilon V\left(y_{*}\right) \beta_{*}+\mathrm{i} \epsilon^{2} V^{\prime}\left(y_{*}\right)-\mathrm{i} \epsilon^{2} \gamma^{2}+\gamma W\left(y_{*}\right),
$$

where $\epsilon=1 / \sqrt{R}$. From a mathematical point of view, the symbol $f\left(y_{*}, \beta_{*}\right)$ is closely related to the eikonal equation in WKBJ theory (Bender \& Orszag 1978). From (4.3) we derive the corresponding twist condition as

$$
\epsilon^{3} V\left(y_{*}\right) V^{\prime \prime}\left(y_{*}\right)+\epsilon 2 \beta_{*}{ }^{2} V^{\prime}\left(y_{*}\right)+2 \beta_{*} \gamma W^{\prime}\left(y_{*}\right)<0 .
$$

From this inequality we can determine the range of $y_{*}$ and $\beta_{*}$ that will produce pseudo-modes with a wave packet shape. Figure 5 shows the range of values $\left\{y_{*}, \beta_{*}\right\}$ for which the twist condition is satisfied. In the free stream, the twist condition is 

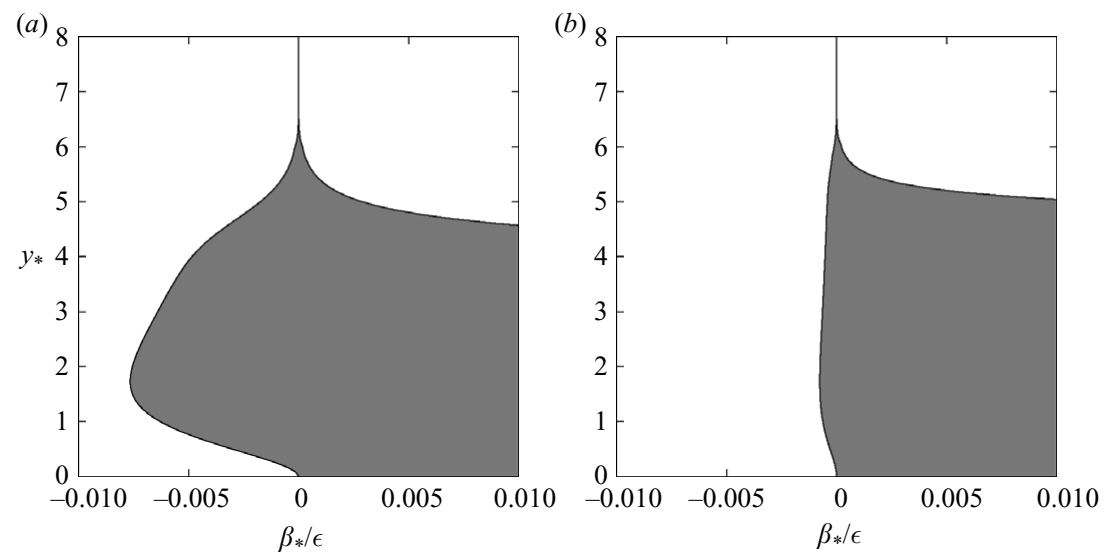

FIgURE 5. The twist condition (4.4) for the uniform swept Hiemenz problem is satisfied outside the shaded region: for $(a) \gamma=0.3, R=100$ and $(b) \gamma=0.3, R=1000$.
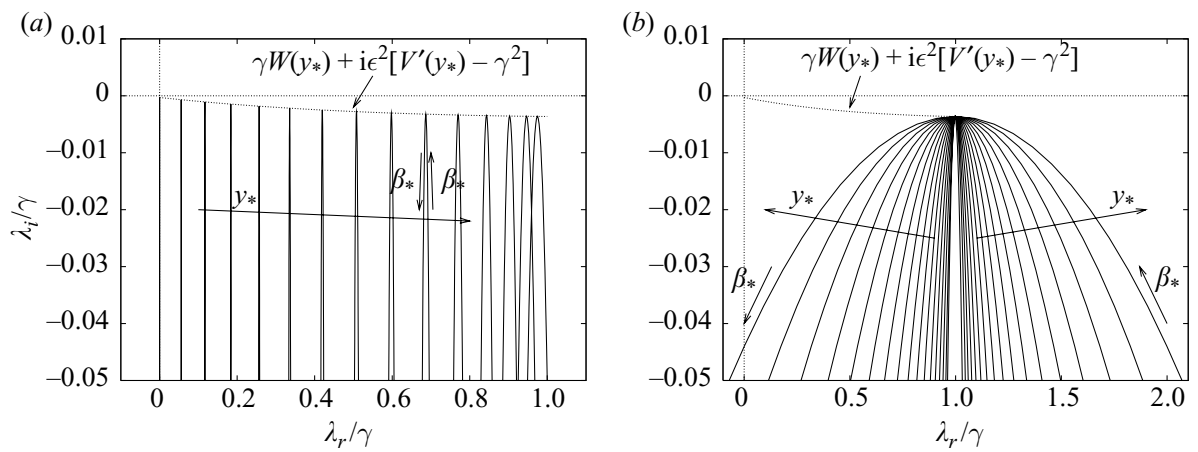

FIGURE 6. Symbol curves $(a)$ within the boundary layer and $(b)$ in the free stream.

satisfied for all wavenumbers $\beta_{*}$ except for a narrow interval near $\beta_{*}=0$. Close to the wall the twist condition is only satisfied for sufficiently negative $\beta_{*}$ (for $\gamma>0$ ), whereas for positive $\beta_{*}$ no wave packet solutions can be found near the wall. In the limit of large Reynolds numbers $(\epsilon \rightarrow 0)$ the region in the $\left(y_{*}, \beta_{*}\right)$-plane where the twist condition is not satisfied protrudes less and less into the left half-plane $\left(\beta_{*}<0\right)$ but extends farther outside the boundary layer. Nevertheless, there always exists an extensive region in the $\left(y_{*}, \beta_{*}\right)$-plane where wave packet pseudo-modes can be found.

Next, we introduce 'symbol curves' as curves in the complex plane, i.e. $\lambda=f\left(y_{*}, \beta_{*}\right)$ for $\beta_{*} \in \mathbb{R}$ with $y_{*}$ fixed to a constant value. For our problem, the symbol curves are parabolas in the complex plane (figure 6) with $\beta_{*}$ parameterizing the curves from right to left and with their apex at $\lambda=\gamma W\left(y_{*}\right)+\mathrm{i} \epsilon^{2}\left[V^{\prime}\left(y_{*}\right)-\gamma^{2}\right]$ (which can be derived readily from (4.3) by setting $\beta_{*}=0$ ). Inside the boundary layer (figure $6 a$ ) the parabolas become wider and move from $\lambda_{r}=0$ towards $\gamma$ as $y_{*}$ increases. Outside the boundary layer (figure $6 b$ ), the parabolas continue to become wider with increasing $y_{*}$, but their apex remains locked at $\gamma-\mathrm{i} \epsilon^{2}\left(1+\gamma^{2}\right)$. Figure 7 displays the entire set of symbol curves. We notice that they cover the entire complex plane below $-\mathrm{i} \epsilon^{2}\left(1+\gamma^{2}\right)$, which corresponds exactly to the upper limit (3.2) for the decay rate $\lambda_{i}$ of algebraically decaying modes. Furthermore, we find that our observations about the location of the peak of the modes from the previous section (cf. figure 4) match the structure of 


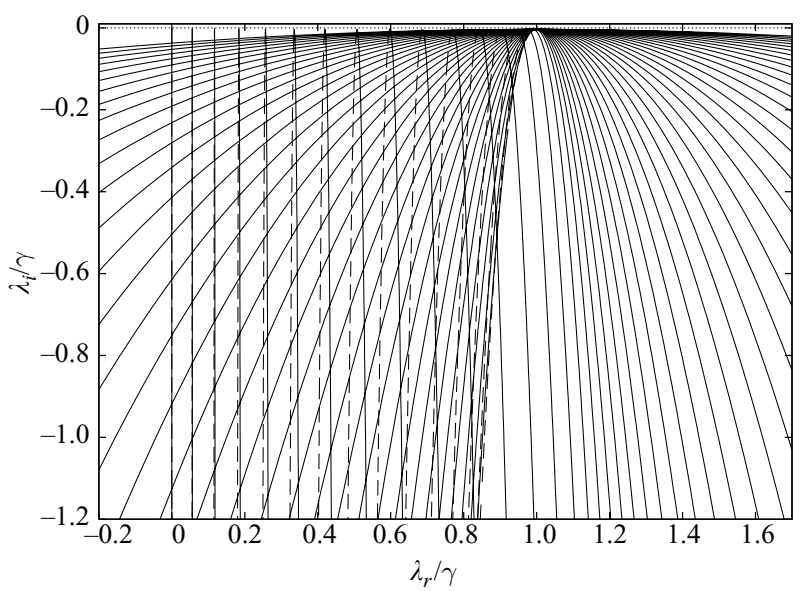

FIGURE 7. Symbol curves for $y_{*}=0, \ldots, 100(\gamma=0.3, R=1000)$. The solid lines indicate that the twist condition is satisfied, whereas the twist condition in not satisfied for the broken lines.

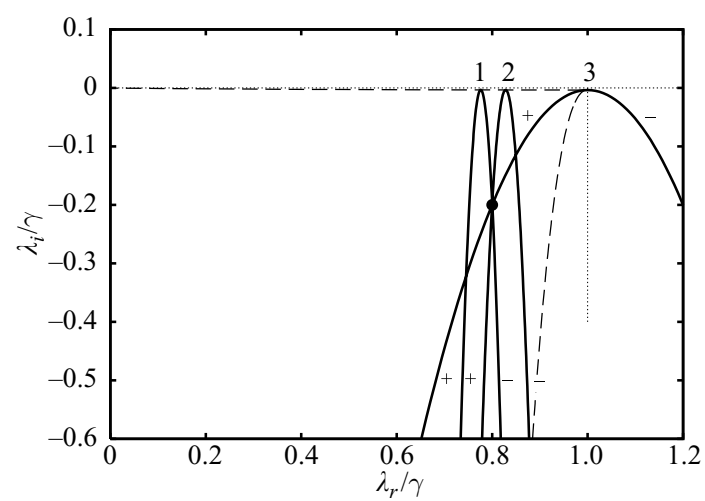

FIGURE 8. Three symbol curves going through a single point $\lambda=(0.8-0.2) / \gamma$. Curves 1 and 2 belong to the boundary layer, and curve 3 is in the free stream. The branches of the symbol curves are denoted by ' + ' (for the left branch with $\beta_{*}>0$ ) and by ' - ' (for the right branch with $\beta_{*}<0$ ). The broken line shows the envelope on the symbol curves (4.4).

the symbol curves; i.e. the wave packets move farther away from the wall as $\left|f_{r}-\gamma\right|$ increases and/or as $f_{i}$ increases (where $\left.f\left(y_{*}, \beta_{*}\right)=f_{r}+\mathrm{i} f_{i}\right)$. This correspondence between the symbol curves and shape of the spectrum leads us to hypothesize that wave packet pseudo-modes, described by (4.3) and (4.4), are approximations to the algebraically decaying eigenmodes for the swept Hiemenz flow. In that same sense, the symbol $f$ is also an approximation to an eigenvalue $\lambda$, and we may use the symbol $f$ as a substitute for an eigenvalue in our analysis of the spectrum. By setting $\lambda=f\left(y_{*}, \beta_{*}\right)$ we define a map between the position $y_{*}$ and local wavenumber $\beta_{*}$ of the wave packet pseudo-mode and the eigenvalue $\lambda$ of the algebraically decaying eigenmode. This map is illustrated by the symbol curves.

Of course, we can also invert this map and look for parameter pairs $\left\{y_{*}, \beta_{*}\right\}$ which correspond to a given $\lambda$. This inverse mapping is not unique but can be described by three separate branches. In other words, a given eigenvalue $\lambda$ can belong to three different symbol curves as shown in figure 8. Symbol curves 1 and 2 describe wave packet pseudo-modes located within the boundary layer, while symbol curve 3 
represents pseudo-modes in the free stream. The parabolic symbol curve 1 passes through a given eigenvalue $\lambda$ with its negative leg, i.e. $\beta_{*}<0$, whereas symbol curves 2 and 3 pass through $\lambda$ with their positive leg $\left(\beta_{*}>0\right)$. Consequently, there can be up to three distinct parameter pairs $\left\{y_{*}, \beta_{*}\right\}$ which yield the same $\lambda=f\left(y_{*}, \beta_{*}\right)$.

To distinguish between the three solutions we define a ' - ' branch (curve 1 ), a ' + bl branch (curve 2 , in the boundary layer) and a ' $+_{f_{s}}$ ' branch (curve 3 , in the free stream). As we can see from figure 7, an eigensolution can simultaneously correspond to the three branches only for $\lambda$ lying in the interval $0 \leqslant \lambda_{r} \lesssim \gamma$. Outside this region, there is a unique association of an eigenmode with either the '-' branch (positive $\lambda_{r}$ ) or with the ' $f_{f_{s}}$ ' branch (negative $\lambda_{r}$ ).

The region in the complex $\lambda$-plane for which we have the coexistence of three solution branches is bounded by the envelope of the respective symbol curves. This envelope is indicated in figure 8 by a broken line. The general formula for an envelope on a one-parameter family of curves $f\left(y_{*}, \beta_{*}\right)$ with the variable $\beta_{*}$ and the 'parameter' $y_{*}$ is given by

which results in

$$
\frac{\partial f_{r}}{\partial \beta_{*}} \frac{\partial f_{i}}{\partial y_{*}}-\frac{\partial f_{r}}{\partial y_{*}} \frac{\partial f_{i}}{\partial \beta_{*}}=0
$$

$$
\epsilon^{3} V\left(y_{*}\right) V^{\prime \prime}\left(y_{*}\right)+\epsilon 2 \beta_{*}^{2} V^{\prime}\left(y_{*}\right)+2 \beta_{*} \gamma W^{\prime}\left(y_{*}\right)=0 .
$$

We observe that the left-hand side of this expression corresponds formally to the twist condition (4.4). This suggests a more detailed investigation of the twist condition. The part of the symbol curves where the twist condition is not satisfied is displayed by broken lines in figure 7 . In our branch nomenclature, it follows that the ' $+_{b l}$ ' branch never satisfies the twist condition, while solutions from the two other branches correspond to actual wave packet pseudo-modes. The same result is also shown in figure 9 , which displays curves of constant $\lambda_{r}$ in the $\left(y_{*}, \beta_{*}\right)$-plane. In the same figure, the thick solid curve indicates the border of the region in which the twist condition is not satisfied, i.e. the region of the ' $+b$ ' branch. We also know from the previous paragraph that this curve corresponds to envelope (4.6) mapped on to the $\left(y_{*}, \beta_{*}\right)$-plane.

In the free stream, we recall (cf. figure 5) that the twist condition is satisfied for all $\beta_{*}$, except for a narrow interval about $\beta_{*}=0$. Close to the wall the twist condition is only satisfied for sufficiently negative $\beta_{*}$ (assuming that $\gamma>0$ ).

In summary, we can conclude that every eigenvalue $\lambda$ which satisfies (3.2) corresponds to at least one wave packet pseudo-mode located in the free stream (either from the ' $f_{s \mathrm{~s}}$ ' branch or from the ' - ' branch) and, in some cases, also to a wave packet pseudo-mode in the boundary layer (from the '-' branch).

\subsection{Algebraically decaying eigenmodes approximated by wave packet pseudo-modes}

In this section, we test our hypothesis that the wave packet pseudo-modes approximate algebraically decaying eigenmodes. To this end, we compare the numerically computed algebraically decaying modes from $\S 3$ with results obtained from the symbol $f\left(y_{*}, \beta_{*}\right)$. This will allow us to confirm that the predictions from the theory of wave packet pseudo-modes are consistent with numerically evaluated eigenfunctions with algebraic decay. In other words, we will show that the wave packet pseudo-modes are as good as actual eigenfunctions.

Figure 10 shows the envelope and the Fourier spectrum of the algebraically decaying mode for $\lambda=(1.5-0.1 \mathrm{i}) \gamma$ (cf. figure $3 a$ ). For this choice of the eigenvalue $\lambda$ only the '-' branch of the symbol yields a wave packet pseudo-mode. The corresponding 


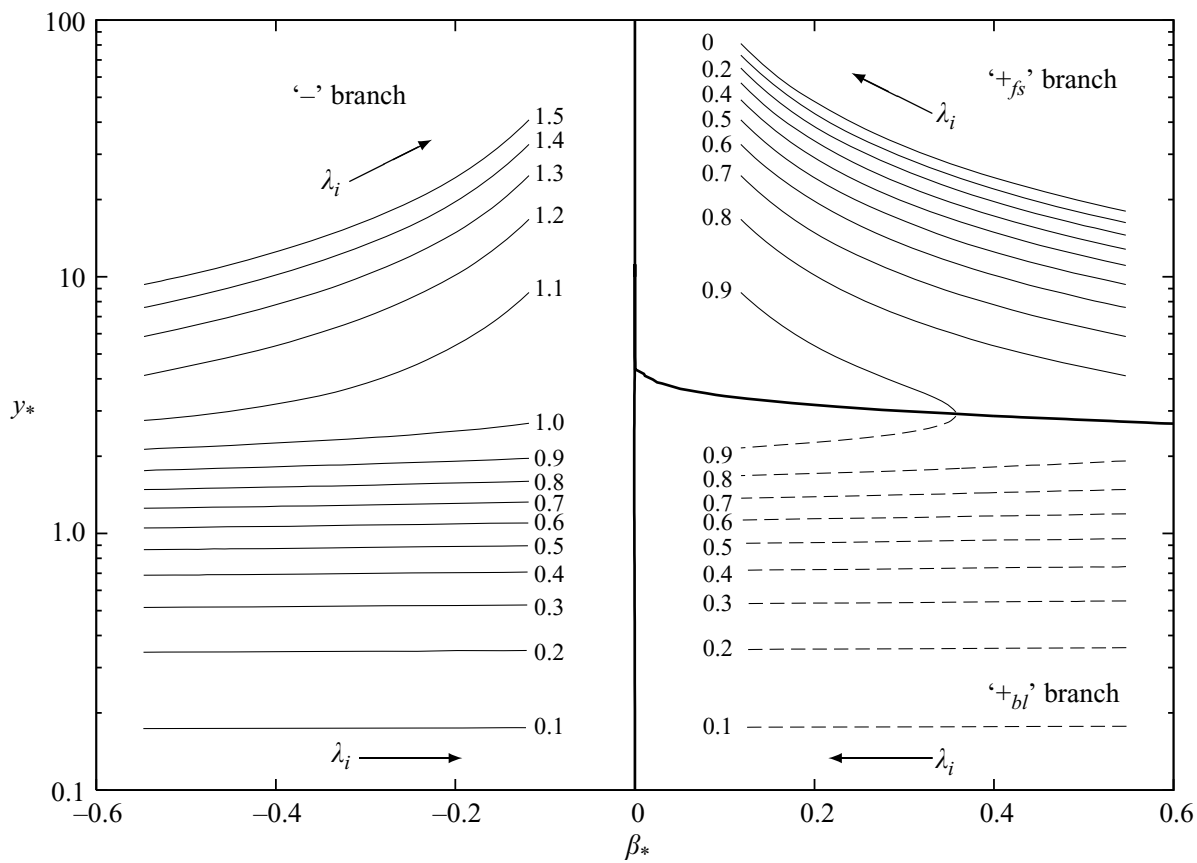

FiguRE 9. Ranges of $\left\{y_{*}, \beta_{*}\right\}$ for which the twist condition is satisfied (' $+f_{s}$ ' and ' - ' branches) or is not satisfied (' $+b l$ branch). The thin lines are curves for $\lambda_{r}=$ const. and $\lambda_{i} / \gamma$ ranging from -1 to -0.05 (the value of $\lambda_{r} / \gamma$ is indicated next to the curves; $\gamma=0.3, R=1000$ ).

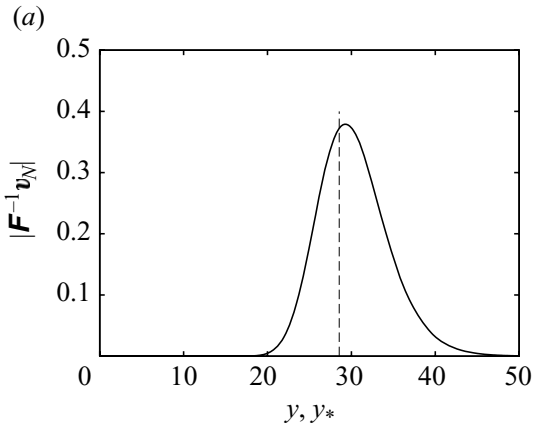

(b)

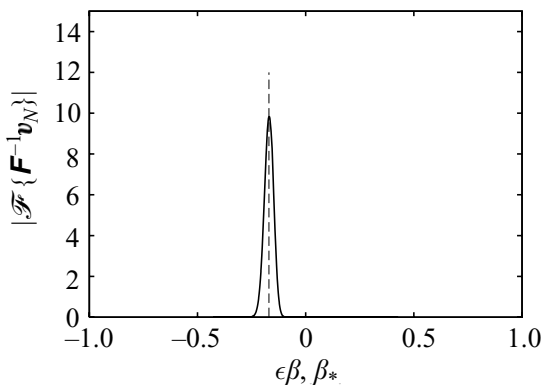

FiguRE 10. (a) Absolute value and $(b)$ Fourier spectrum of the algebraically decaying mode for $\lambda=(1.5-0.1 \mathrm{i}) \gamma$ together with the values $y_{*}$ and $\beta_{*}$ from the '-' branch (broken lines) for which $\lambda=f\left(y_{*}, \beta_{*}\right)$.

location $y_{*}$ and local wavenumber $\beta_{*}$, obtained from the inverse symbol map, are indicated by the broken lines. We observe that the shape of this mode is predicted rather well by the symbol (even though the actual peak location is somewhat further to the right than predicted).

The interpretation for the eigenvalue $\lambda=(0.7-0.4 \mathrm{i}) \gamma$ (cf. figure $3 b)$ is somewhat more involved. In this case, the symbol attains this eigenvalue for three pairs of $\left\{y_{*}, \beta_{*}\right\}$ as indicated in figure 11. The associated mode features two wave packets which correspond to $\left\{y_{*}, \beta_{*}\right\}$ from the '-' branch (for the wave packet in the boundary layer) and to $\left\{y_{*}, \beta_{*}\right\}$ from the ' $+_{f_{s}}$ ' branch (for the wave packet in the free stream). 

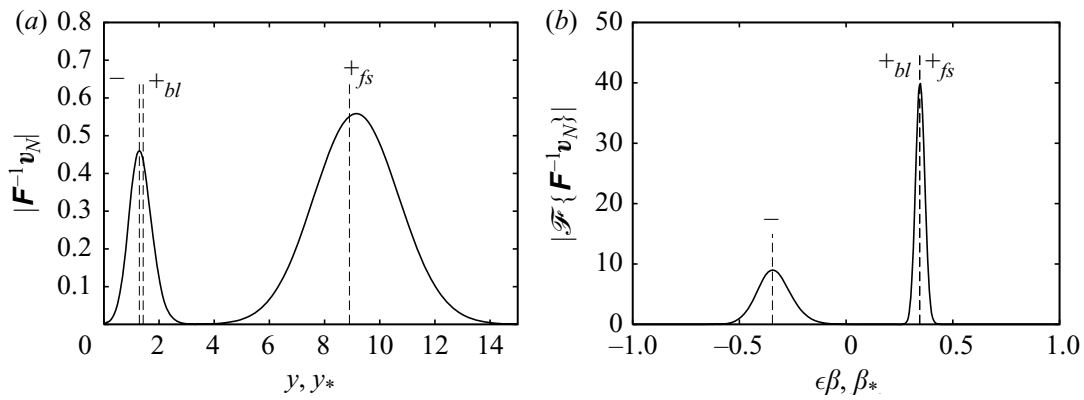

Figure 11. (a) Absolute value and $(b)$ Fourier spectrum of the algebraically decaying mode for $\lambda=(0.7-0.4 \mathrm{i}) \gamma$ together with the values $y_{*}$ and $\beta_{*}$ (broken lines) for which $\lambda=f\left(y_{*}, \beta_{*}\right)$ (for the ' $t_{b l}$ ' and the ' $+_{f_{s}}$ ' branches the wavenumbers $\beta_{*}$ are nearly equal).

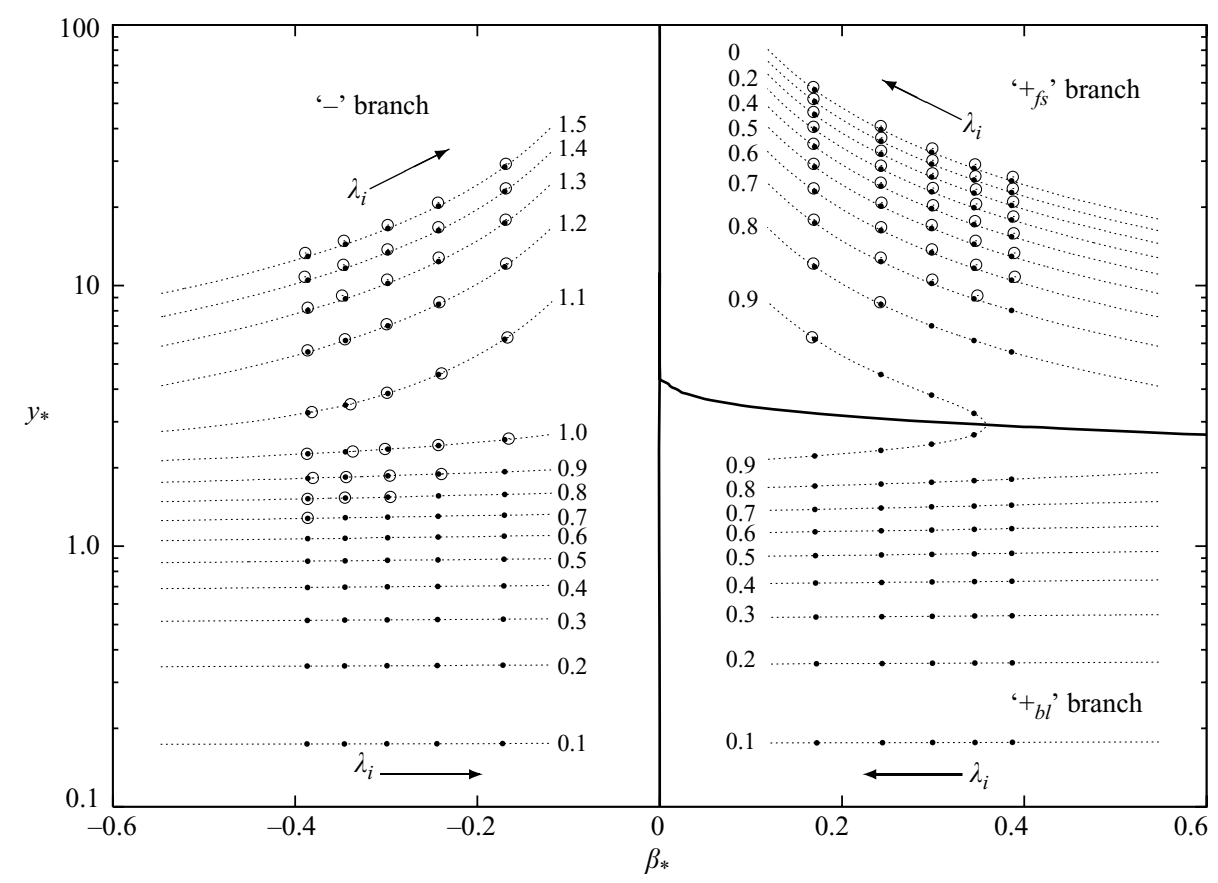

FIGURE 12. Location of the peak and the wavenumber of the numerically evaluated wave packet pseudo-mode ( $\circ$ ) compared to the values predicted by the symbol $(\bullet)$. The dotted curves are lines for which $\lambda_{r}$ is constant (the value of $\lambda_{r} / \gamma$ is indicated next to the curves). The dots indicate different values of the imaginary part $\lambda_{i} / \gamma=-0.5,-0.4,-0.3,-0.2,-0.1$. The solid line defines the border of the region for which the twist condition is satisfied $(\gamma=0.3, R=1000)$.

There is no wave packet associated with the ' + bl branch that would be consistent with the twist condition.

We have repeated such tests for a larger number of eigenvalues and have summarized the results in figure 12, which compares the predictions from the symbol to the measured peak location and dominant wavenumber of the algebraically decaying eigenmode. 


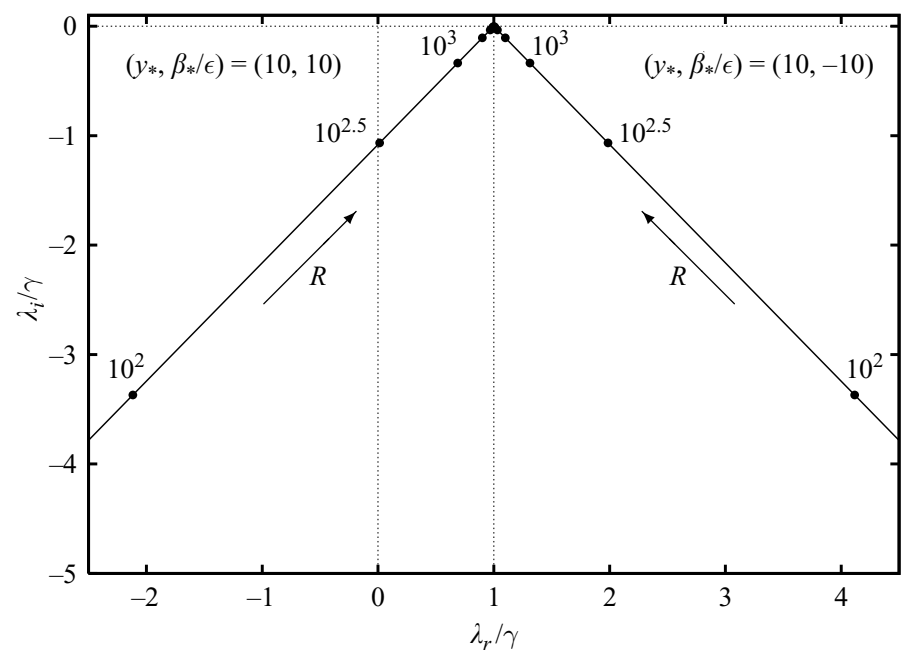

FigURE 13. Estimated eigenvalues $\lambda$ for eigenfunctions with a wave packet at $y_{*}=10$ with wavenumber $\beta_{*} / \epsilon=-10$ (right branch) or with wavenumber $\beta_{*} / \epsilon=10$ (left branch). The Reynolds number changes from $R=10$ (not visible) to $R=10^{5}(\gamma=0.3)$.
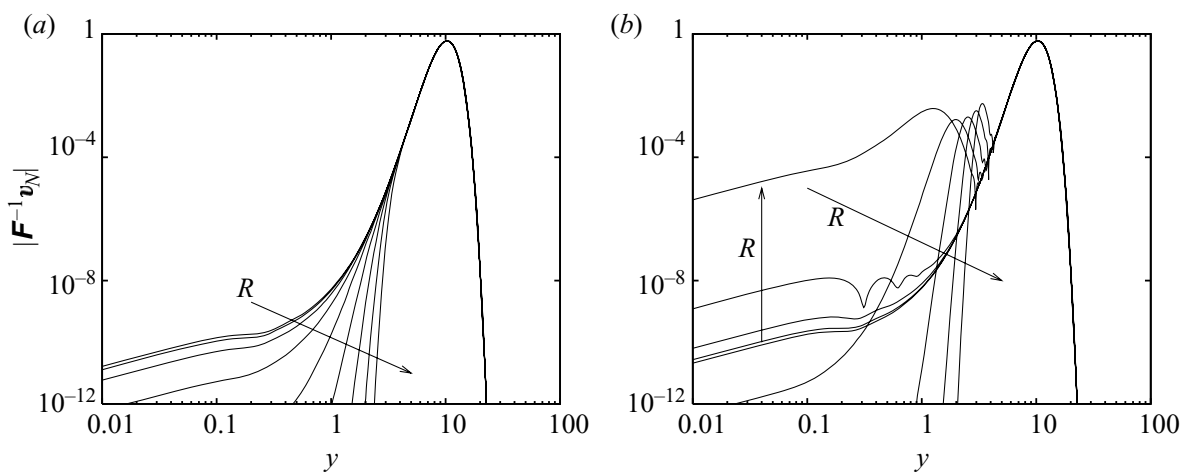

Figure 14. Absolute value of the eigenfunctions for a wave packet at $y_{*}=10$ with (a) $\beta_{*} / \epsilon=-10$ and $(b) \beta_{*} / \epsilon=10$ for the Reynolds numbers $R=10,10^{1.5}, 10^{2}, 10^{2.5}, 10^{3}$, $10^{3.5}, 10^{4}, 10^{4.5}, 10^{5}(\gamma=0.3)$.

\subsection{Influence of the Reynolds number}

So far, our results were computed for a Reynolds number $R=1000$. This section is devoted to the effect of the Reynolds number on the algebraically decaying modes. To this end, we vary the Reynolds number from $R=10$ to $R=10^{5}$ and determine the algebraically decaying eigenfunctions which exhibit wave packets at $y_{*}=10$ with wave numbers $\beta_{*} / \epsilon=10$ and $\beta_{*} / \epsilon=-10$. (The spanwise wavenumber $\gamma$ is maintained at 0.3.) The symbol (4.3) gives us an estimate of the eigenvalue $\lambda$ associated with these eigenfunctions (figure 13). We then use the procedure outlined in $\S 3$ to compute the eigenfunctions $\boldsymbol{F}^{-1} \boldsymbol{v}_{N}$ (figure 14). Surprisingly, we observe that the shape of the eigenfunctions in the free stream are almost independent of the Reynolds number. For $\beta_{*} / \epsilon=-10$, the differences inside the boundary layer are small. For $\beta_{*} / \epsilon=10$, however, the situation is a bit more complex inside the boundary layer. Figure 13 shows that Reynolds numbers less than approximately $10^{2.5}$ result in eigenvalues with 

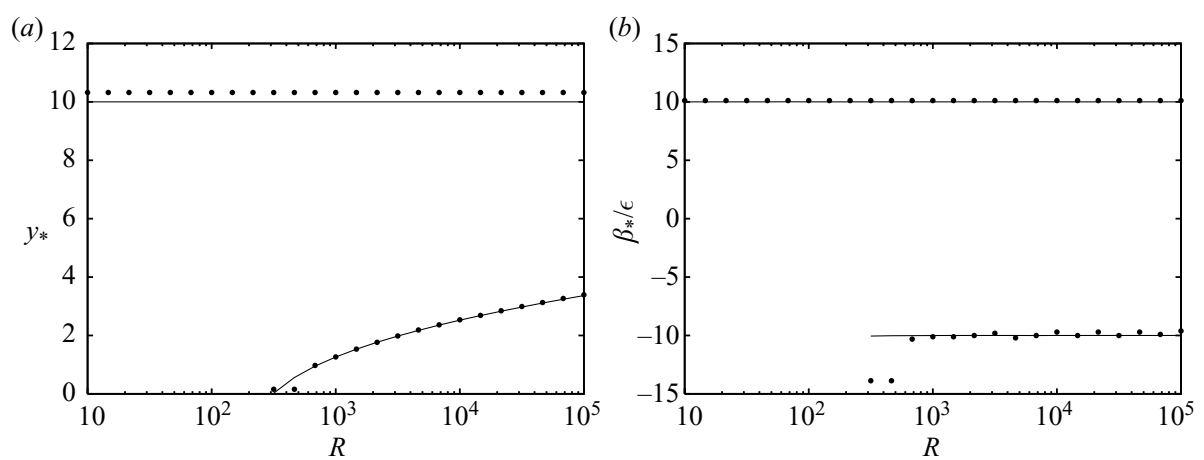

FIGURE 15. Comparison between the algebraically decaying modes $(\bullet)$ and the predictions ( $\longrightarrow$ ) from the symbol for $y_{*}=10, \beta_{*} / \epsilon=10$ and $\gamma=0.3:(a)$ location(s) and (b) dominant wavenumber(s) of the wave packet(s).

negative real part. For such eigenvalues the symbol allows only one wave packet solution from the ' $f_{f s}$ ' branch in the free stream. For Reynolds numbers larger than approximately $10^{2.5}$ we observe two wave packets ('-' and ' $+_{f_{s}}$ ' branches). The emergence of two wave packets is clearly visible in figure 14(b).

Finally, figure 15 compares the location and the dominant wavenumber(s) of the wave packet(s) with the predictions from the symbol. It confirms that the symbol yields good approximations for the shape and temporal behaviour of algebraically decaying eigenfunctions. Appreciable differences between algebraically decaying modes and the symbol can only be found for small Reynolds numbers. We have observed similar results with respect to the parameter $\gamma$, namely an insensitivity of the shape of the wave packet in the free stream. However, these results are omitted in this paper.

To conclude, a link between algebraically decaying modes and wave packet pseudomodes has been established. Together with the asymptotic results (3.4a) and (3.4b) for $y \rightarrow \infty$, the symbol (4.3) and the twist condition (4.4) yield excellent estimates for the shape of an algebraically decaying eigenfunction associated with a given eigenvalue $\lambda$. We have also seen that the shapes of algebraically decaying eigenmodes in the free stream are (nearly) independent of the Reynolds number and of the spanwise wavenumber $\gamma$. And lastly, the symbol curves support our hypothesis that the spectrum of the algebraically decaying modes fills the whole complex half-plane bounded by (3.2).

\section{The role of algebraically decaying modes}

In the previous sections, we have analysed and discussed the eigenfunctions and the eigenvalue spectrum of the algebraically decaying modes from a theoretical point of view without regard to their physical role in the swept Hiemenz flow. The following sections will address this role and demonstrate the relevance of algebraically decaying modes on a few selected examples covering single and standing waves as well as travelling wave packets.

In $\S 5.1$ and 5.2, we study the flow field of algebraically decaying modes. Although these modes are not found in an isolated form in experimental investigations, they are needed to describe the full dynamics of the flow. Similar to the modes of the continuous spectrum of the Blasius boundary layer (Grosch \& Salwen 1978; Salwen \& Grosch 1981) the algebraically decaying modes are found in the flow field outside the 
boundary layer. The super-exponentially decaying modes decay too rapidly to play a major role in this region. In contrast to the continuous modes of Salwen \& Grosch (1981), the algebraically decaying modes have (asymptotically) compact support and are parameterized by their wave number and by their location, which results in an area-filling continuous spectrum. This difference is directly related to the character of the respective eigenvalue problems: in the free stream of the Blasius boundary layer the Orr-Sommerfeld equation becomes a differential equation with constant coefficients, which leads to exponential solutions, while the swept stagnation point flow outside the leading-edge boundary layer leads to an eigenvalue problem with variable coefficients, which supports wave packet pseudo-modes (Trefethen \& Embree 2005).

In the present investigation, we consider the swept Hiemenz flow. For $y \rightarrow \infty$, this flow blends into a swept stagnation point flow to which we refer as the 'free stream'. However, in reality (e.g. for a swept cylinder) this stagnation point flow transitions smoothly into a parallel flow at $y=O(R)$. Therefore, the swept stagnation point flow represents an interior layer between the boundary layer and the actual free stream. Similar to the free stream outside the Blasius boundary layer, the parallel flow upstream of the Hiemenz flow does not support wave packet pseudo-modes. Therefore, the transition from stagnation point flow to the free stream has a direct effect on the symbol curves and the twist condition. This issue has been discussed in detail by Obrist \& Schmid (2009), where it is shown that the algebraic decay in the interior layer is followed by an exponential decay in the free stream. Because of the strong decay in the free stream, it can be shown that this flow configuration even allows for modes with moderate algebraic growth in the interior layer, which is consistent with the arguments of Dhanak \& Stuart (1995).

The wave packet pseudo-modes in the stagnation point flow are important for the communication between the free stream and the boundary layer. They are a central component of a two-step receptivity process: $(a)$ free-stream perturbations are matched to algebraic modes which transport the perturbations towards the wall, (b) where they trigger boundary layer instabilities. In $\S 5.3$, we demonstrate how the theory of wave packet pseudo-modes can be used to study this transport towards the boundary layer.

\subsection{Radiating and incoming oblique waves}

We will start by considering a single algebraically decaying mode. According to ansatz (2.1) the two modes shown in figure 3, for example, lead to a uniform disturbance field $u(x, y, z)$ of the form

$$
u(x, y, z)=\hat{u}(y) \mathrm{e}^{\mathrm{i}\left(\gamma z-\lambda_{r} t\right)+\lambda_{i} t} .
$$

Snapshots of these disturbance fields are shown in figure 16. We see that the wave packets represented by the eigenfunctions result in oblique waves in the $(y, z)$-plane, which are modulated by a stationary envelope function. From (5.1) it follows that these waves travel under the envelope in the $z$-direction with a phase velocity of $\lambda_{r} / \gamma$. Since the wave packets contain waves with wavenumbers $\beta_{*} / \epsilon$ we can determine the local phase velocity in the $y$-direction as $\epsilon \lambda_{r} / \beta_{*}$, which yields an oblique wave propagating in the direction of the wavenumber vector $\left(\gamma, \beta_{*} / \epsilon\right)$ with phase velocity

$$
c_{p}=\frac{\lambda_{r}}{\sqrt{\gamma^{2}+\left(\beta_{*} / \epsilon\right)^{2}}} .
$$



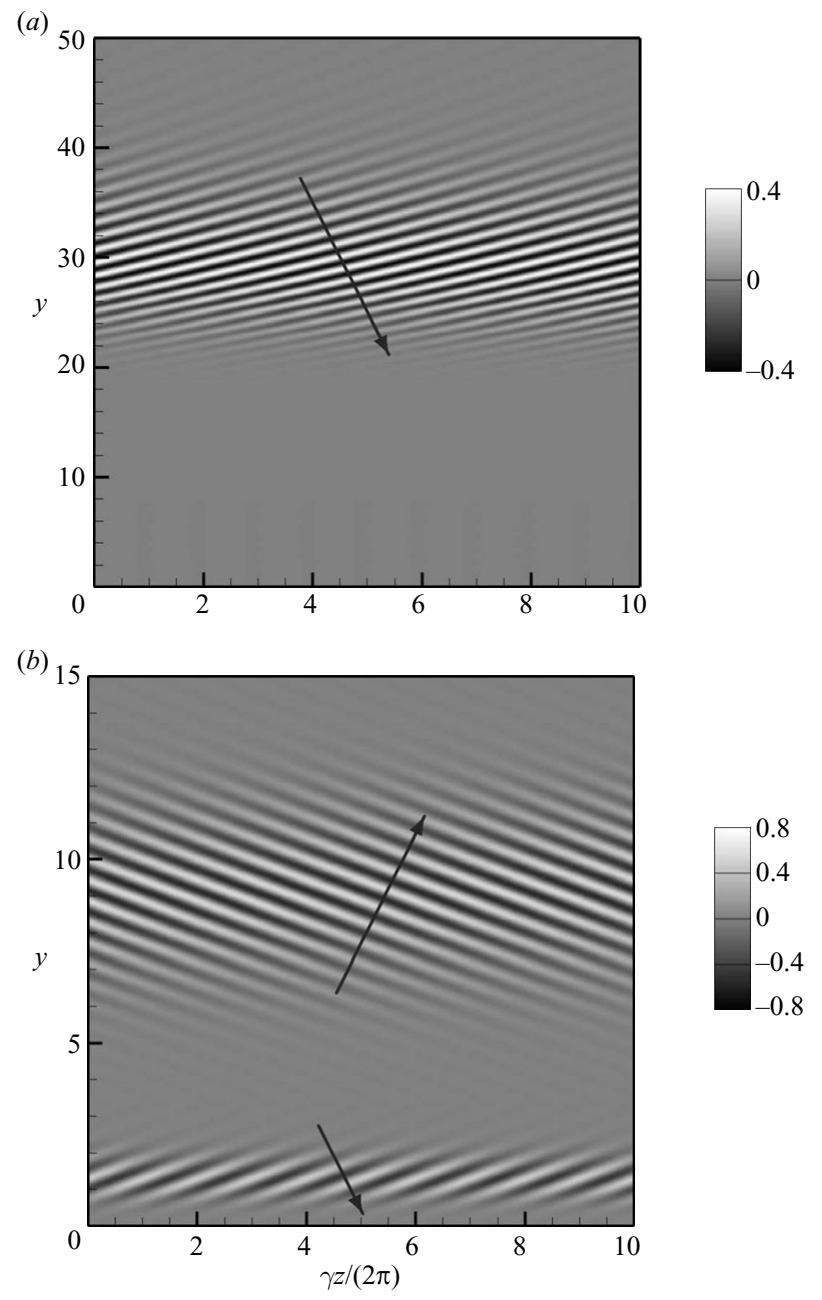

FIGURE 16. Disturbance fields of the algebraically decaying eigenmodes for $(a) \lambda=(1.5-0.1 i) \gamma$ and $(b) \lambda=(0.7-0.4 \mathrm{i}) \gamma$. The arrows indicate the dominant direction of the wave propagation. $(\gamma=0.3, R=1000)$.

Waves of this type move towards the wall for $\beta_{*}<0$ and radiate away from the wall for $\beta_{*}>0$ (for $\lambda_{r}>0$ ). The dominant directions of propagation are indicated by arrows in figure 16, and a comparison with figures $10(b)$ and $11(b)$ shows that the propagation directions are in agreement with $\beta_{*}$.

The local wave propagation within the wave packets is summarized in figure 17. It illustrates the direction and phase velocity $c_{p}$ for different eigenvalues $\lambda$. Depending on the twist condition this plot indicates the wave propagation for a single wave packet pseudo-mode in the free stream or for two wave packets, one in the boundary layer and one in the free stream. Note that waves in the boundary layer can only propagate towards the wall.

\subsection{Standing wave packets in the free stream}

The observation that the local phase velocity in the $y$-direction is proportional to $\beta_{*}$ suggests the possibility of standing waves by superimposing two algebraically decaying 


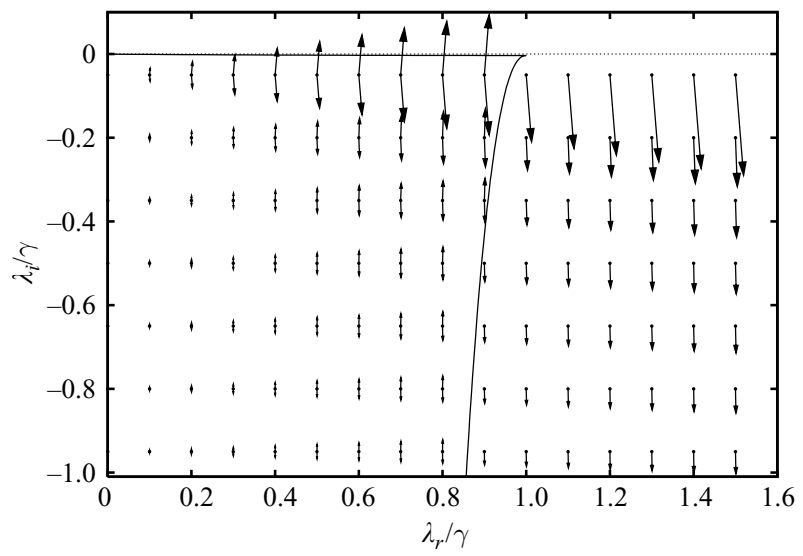

FIGURE 17. Direction and magnitude of the phase velocity of the local wave propagation as a function of the eigenvalue $\lambda(\gamma=0.3, R=1000)$. The arrows are plotted as if they were in the $(z, y)$-plane (cf. figure 16). The solid line indicates the twist condition.

waves with $\pm \beta_{*}$. The attribute 'standing' refers in this case to the propagation in the $y$-direction. A similar idea was proposed by Obrist (2000) for the super-exponentially decaying modes in the swept Hiemenz flow. We use two modes from the same symbol curve with wavenumbers of opposite signs, $\beta_{*, 1}=-\beta_{*, 2}$. Both modes have the identical decay rate $\lambda_{i}$ because of the symmetry of the parabolic symbol curve. The superposition of such two modes (figure 18a) leads to standing waves in $y$, which are advected in the $z$-direction with the free-stream sweep velocity. Apart from the standing wave packet in the free stream there also exists an oblique wave packet inside the boundary layer that belongs to the '-' branch (cf. figure 16b).

It is worth mentioning that as a special case, there also exist standing wave packets for $\lambda_{r}=0$. These wave packets (figure $18 b$ ) are stationary and do not even propagate in the $z$-direction; they only decay according to $\exp \left(\lambda_{i} t\right)$.

\subsection{Travelling wave packets in the free stream}

So far, we have only considered single modes or superpositions of two modes with the same decay rate. Accordingly, the overall shape of the flow field did not change except for a decay of the amplitude. We will now study a superposition of many modes to describe travelling wave packets in the free stream. It is important to clarify in this context that we must not confuse these travelling wave packets with the wave packets found for the algebraically decaying modes. The latter modal wave packets maintain their position along the $y$-axis at all times, whereas the former travelling wave packets will propagate towards the wall. They represent travelling localized disturbances consisting of a superposition of modal solutions.

We start by describing a local initial perturbation in the form of a wave packet at $y_{0}$ with wavenumber $\beta_{0}$ by its Fourier integral,

$$
u_{0}(y)=A \mathrm{e}^{\mathrm{i} \beta_{0}\left(y-y_{0}\right)} \mathrm{e}^{-\left(y-y_{0}\right)^{2} /(2 \sigma)^{2}}=A \frac{\sigma}{\sqrt{\pi}} \int_{-\infty}^{\infty} \mathrm{e}^{-\left(\beta-\beta_{0}\right)^{2} \sigma^{2}} \mathrm{e}^{\mathrm{i} \beta\left(y-y_{0}\right)} \mathrm{d} \beta .
$$

The dynamics of this initial condition will be governed by the dynamics of modes near $y_{0}$ with wavenumbers close to $\beta_{0}$. We recall from the previous sections that the dynamics of such modes is (approximately) governed by the symbol $f(y, \beta \epsilon)$. 

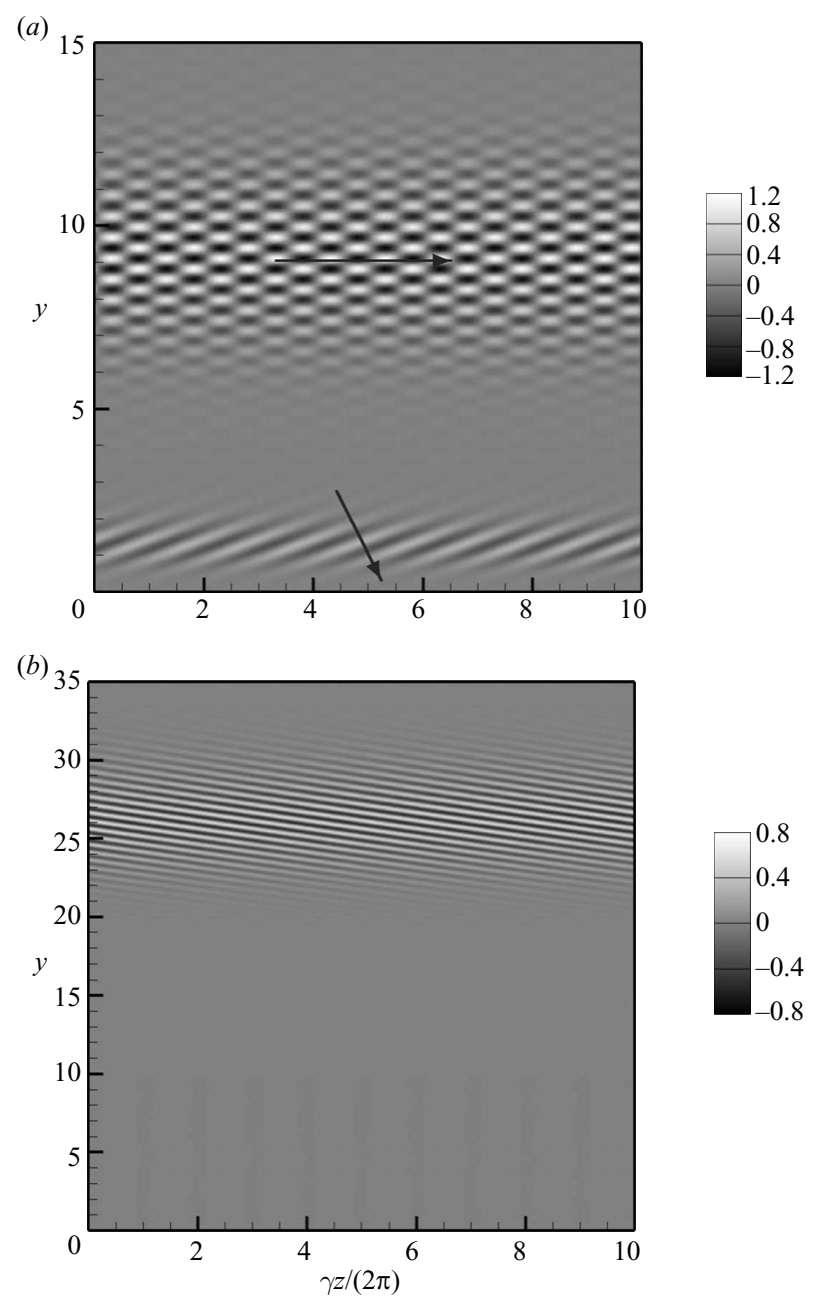

FIGURE 18. Disturbance fields of $(a)$ a superposition of the algebraically decaying eigenmodes for $\lambda=((1 \pm 0.3-0.4 \mathrm{i}) \gamma$ and $(b)$ the eigenmodes for $\lambda=(0-0.5 \mathrm{i}) \gamma$. The arrows indicate the dominant direction of the wave propagation $(\gamma=0.3, R=1000)$.

Therefore, we approximate the evolution of the flow field by

$$
u(y, t) \approx A \frac{\sigma}{\sqrt{\pi}} \int_{-\infty}^{\infty} \mathrm{e}^{-\left(\beta-\beta_{0}\right)^{2} \sigma^{2}} \mathrm{e}^{\mathrm{i} \beta\left(y-y_{0}\right)-\mathrm{i} f(y, \beta \epsilon) t} \mathrm{~d} \beta .
$$

We can evaluate this integral expression by expanding the symbol about $\beta_{0}$,

$$
f(y, \beta \epsilon) \approx f\left(y, \beta_{0}\right)+\left(\beta \epsilon-\beta_{0}\right) c_{r}+\mathrm{i}\left(\beta \epsilon-\beta_{0}\right) c_{i},
$$

where

$$
c_{r}=\operatorname{Re}\left\{\left.\frac{\partial f}{\partial \beta_{*}}\right|_{\beta_{*}=\epsilon \beta_{0}}\right\}, \quad c_{i}=\operatorname{Im}\left\{\left.\frac{\partial f}{\partial \beta_{*}}\right|_{\beta_{*}=\epsilon \beta_{0}}\right\} .
$$


We substitute this expansion into (5.4) and obtain (after regrouping terms)

$$
u(y, t) \approx A \mathrm{e}^{-\mathrm{i} f\left(y_{0}, \beta_{0} \epsilon\right) t} \frac{\sigma}{\sqrt{\pi}} \int_{-\infty}^{\infty} \mathrm{e}^{-\left(\beta-\beta_{0}\right)^{2} \sigma^{2}+\left(\beta-\beta_{0}\right) \epsilon c_{i} t} \mathrm{e}^{\mathrm{i} \beta\left[y-\left(y_{0}+\epsilon c_{r} t\right)\right]} \mathrm{d} \beta .
$$

By completing the square in the first exponent of the integral,

$$
-\left(\beta-\beta_{0}\right)^{2} \sigma^{2}+\left(\beta-\beta_{0}\right) \epsilon c_{i} t=-\left[\beta-\left(\beta_{0}+\epsilon c_{i} t /\left(2 \sigma^{2}\right)\right)\right]^{2} \sigma^{2}+\epsilon^{2} c_{i}^{2} t^{2} /\left(4 \sigma^{2}\right),
$$

we can rewrite the Fourier integral as

$$
u(y, t) \approx A \mathrm{e}^{-\mathrm{i} f\left(y_{0}, \beta_{0} \epsilon\right) t+\epsilon^{2} c_{i}^{2} t^{2} /\left(4 \sigma^{2}\right)} \frac{\sigma}{\sqrt{\pi}} \int_{-\infty}^{\infty} \mathrm{e}^{-\left[\beta-\left(\beta_{0}+\epsilon c_{i} t /\left(2 \sigma^{2}\right)\right)\right]^{2} \sigma^{2}} \mathrm{e}^{\mathrm{i} \beta\left[y-\left(y_{0}+\epsilon c_{r} t\right)\right]} \mathrm{d} \beta .
$$

This integral now describes a wave packet centred at $y_{0}+\epsilon c_{r} t$ and localized about the wavenumber $\beta_{0}+\epsilon c_{i} t /\left(2 \sigma^{2}\right)$. The amplitude of this wave packet is governed by the exponential expression in front of the integral. At this point, it is important to emphasize that expression (5.9) holds only for $t \rightarrow 0^{+}$, since it has been derived for a wave packet located at $y_{0}$ with wavenumber $\beta_{0}$. In other words, after an infinitesimally short time $\mathrm{d} t$ the wave packet will have shifted to $y_{0}+\epsilon c_{r} \mathrm{~d} t$ and a new dominant wave number $\beta_{0}+\epsilon c_{i} \mathrm{~d} t /\left(2 \sigma^{2}\right)$. Over the same time interval the amplitude $A$ will have changed by $A \operatorname{Im}\left\{f\left(y_{0}, \beta_{0}\right\} \mathrm{d} t\right.$. This shift in the position and in the wavenumber requires a re-evaluation of $f, c_{r}$ and $c_{i}$.

Summarizing these results, we can say that a wave packet at a position $y_{0}$ with wavenumber $\beta_{0}$ will move with the (group) velocity $c_{g}$ given by

$$
c_{g} \equiv \frac{\mathrm{d} y}{\mathrm{~d} t}=\epsilon \operatorname{Re}\left\{\left.\frac{\partial f}{\partial \beta_{*}}\right|_{y_{*}=y_{0}, \beta_{*}=\epsilon \beta_{0}}\right\},
$$

while its wavenumber will shift by

$$
c_{\beta} \equiv \frac{\mathrm{d} \beta}{\mathrm{d} t}=\frac{\epsilon}{2 \sigma^{2}} \operatorname{Im}\left\{\left.\frac{\partial f}{\partial \beta_{*}}\right|_{y_{*}=y_{0}, \beta_{*}=\epsilon \beta_{0}}\right\}
$$

and its amplitude $A$ will change by

$$
\frac{\mathrm{d} A}{\mathrm{~d} t}=A \operatorname{Im}\left\{f\left(y_{0}, \epsilon \beta_{0}\right)\right\} .
$$

For our flow configuration, we find

$$
\begin{aligned}
c_{g} & =V\left(y_{0}\right) / R, \\
c_{\beta} & =-\beta_{0} /\left(\sigma^{2} R\right), \\
\mathrm{d} A / \mathrm{d} t & =-A\left(\beta_{0}^{2}+\gamma^{2}-V^{\prime}\left(y_{0}\right)\right) / R,
\end{aligned}
$$

where we have replaced $\epsilon^{2}$ again by $1 / R$. We have tested these theoretical predictions by comparing them to the results from a direct numerical simulation of the evolution of the localized perturbation (5.3). Figure 19 shows results for perturbations initially located at $y_{0}=40$ with various widths $\sigma$ and wavenumbers $\beta_{0}$. As predicted by (5.13) we observe that the group velocity $c_{g}$ is nearly independent of $\beta_{0}$ and $\sigma$, that the relative wavenumber shift $c_{\beta} / \beta_{0}$ is nearly independent of $\beta_{0}$ and that the decay rate is nearly independent of $\sigma$. These results indicate that expressions (5.13) are reasonably good predictions for the dynamics of a localized perturbation in the free stream of our flow configuration. The differences between the numerical and the theoretical results can be attributed to the higher-order terms neglected in the Taylor expansion (5.5). 

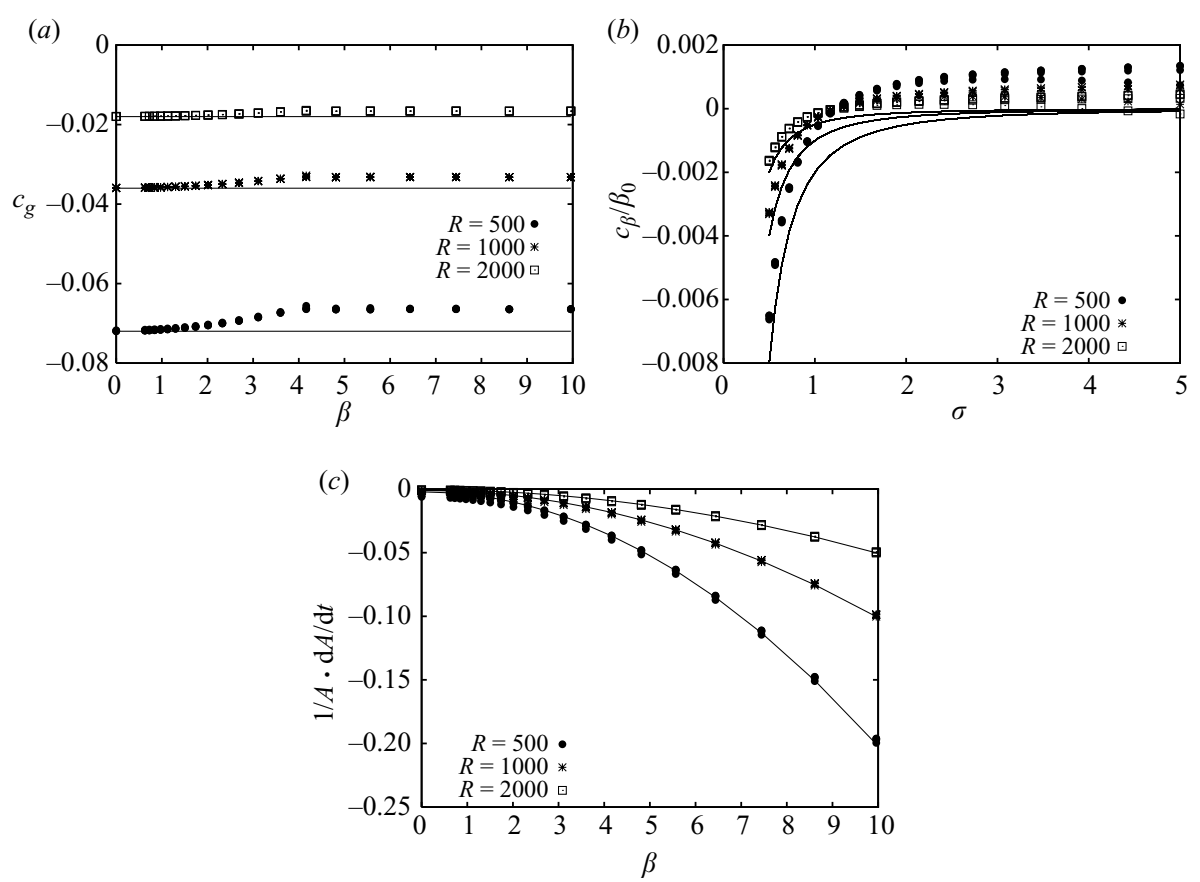

Figure 19. (a) Group velocity $c_{g},(b)$ wavenumber shift $c_{\beta}$ and $(c)$ growth rate for a localized perturbation at $y_{0}=40$ with $\beta_{0}=0, \ldots, 10$ and $\sigma=0.5, \ldots, 5$. (The symbols indicate numerical results, and the solid lines are theoretical predictions.)

\section{Summary and concluding remarks}

For the flow in the vicinity of swept attachment-line boundary layers, modelled by the swept Hiemenz flow, the class of algebraically decaying solutions has been readily identified by several authors, but their role in describing the overall disturbance dynamics has remained unclear. Focusing on the uniform modes of the swept Hiemenz problem - the most accessible model displaying algebraically decaying solutions - this class of solutions has been analysed using the theory of wave packet pseudo-modes. This theory, closely related to a WKBJ approximation of the governing equation, yields approximations to the dispersion relation in algebraic form given by the symbol. The corresponding approximate eigenfunctions (or pseudo-modes) take on the form of wave packets if an additional constraint, the twist condition, is satisfied. This procedure parameterizes the spectrum consisting of algebraically decaying modes by the location and local wavenumber of the associated wave packet and thus allows an efficient analysis of its role and contribution to the overall perturbation dynamics.

Three distinct branches of the algebraically decaying spectrum have been identified corresponding to wave packets in the free stream and/or the boundary layer. The existence of these solutions has been verified by contrasting them with numerical solutions of the initial-value problem.

The analysis has verified the proposition that the swept Hiemenz flow is characterized by a continuous spectrum that consists of a half-plane in the complex plane limited by an upper bound for the imaginary part of the eigenvalues. This area, which can be described by the presented theory, consists of localized solutions that show algebraic decay in the wall-normal direction. As that, they can be thought of as equivalent to the oscillatory solutions in the free stream that form the continuous 
spectrum of a Blasius boundary layer (Grosch \& Salwen 1978; Salwen \& Grosch 1981).

The superposition of modes from the continuous spectrum results in wavefronts and wave packets that depending on their composition, propagate towards or away from the boundary layer or represent standing (but decaying) wave solutions. Their physical role is thus in the communication between free-stream disturbances and boundary layer perturbations. Consequently, they are expected to play an important role in receptivity studies of swept attachment-line boundary layers.

This connecting role between the free stream and the boundary layer cannot be affected by the continuous line spectrum of the super-exponentially decaying modes, since an analysis along $\S 5.3$ reveals that this type of modes does not yield any travelling wave packets but rather standing waves which decay like $\exp (-2 t / R)$. The full dynamic exchange of information between the free stream and the boundary layer thus falls on to the continuous spectrum of algebraically decaying modes.

On a more technical note, the approximation of localized solutions to stability problems by wave packet pseudo-modes, as introduced above, has proven a valuable technique in analysing parts of the full spectrum related to free-stream modes and has helped quantify the relevance of the continuous spectrum as well as its physical importance for receptivity studies.

\section{REFERENCES}

Bender, C. M. \& Orszag, S. A. 1978 Advanced Mathematical Methods for Scientists and Engineers. McGraw-Hill.

BrattKus, K. \& Davis, S. H. 1991 The linear stability of plane stagnation-point flow against general disturbances. Quart. J. Mech. appl. Math. 44 (1), 135-146.

DhanaK, M. R. \& StUART, J. T. 1995 Distortion of the stagnation-point flow due to cross-stream vorticity in the external flow. Phil. Trans. R. Soc. Lond. A 352, 443-452.

GöRTLER, H. 1941 Instabilität laminarer Grenzschichten an konkaven Wänden gegenüber gewissen dreidimensionalen Störungen. Z. Angew. Math. Mech. 21, 250.

Grosch, C. E. \& Salwen, H. 1978 The continuous spectrum of the Orr-Sommerfeld equation. Part 1. The spectrum and the eigenfunctions. J. Fluid Mech. 87, 33-54.

Hall, P. \& MaLiK, M. R. 1986 On the instability of a three-dimensional attachment-line boundary layer: weakly nonlinear theory and a numerical approach. J. Fluid Mech. 163, 257-282.

Hall, P., Malik, M. R. \& Poll, D. I. A. 1984 On the stability of an infinite swept attachment line boundary layer. Proc. R. Soc. Lond. A 395, 229-245.

Hämmerlin, G. 1955 Zur Instabilitätstheorie der ebenen Staupunktströmung. In 50 Jahre Grenzschichtforschung (ed. H. Görtler \& W. Tollmien), p. 315. Vieweg.

Kestin, J. \& Wood, R. T. 1970 On the stability of a two-dimensional stagnation flow. J. Fluid Mech. 44, 461-479.

LiN, R.-S. \& MaLIK, M. R. 1996 On the stability of attachment-line boundary layers. Part 1. The incompressible swept Hiemenz flow. J. Fluid Mech. 311, 239-255.

Lyell, M. J. \& Huerre, P. 1985 Linear and nonlinear stability of plane stagnation flow. J. Fluid Mech. 161, 295-312.

OвRIST, D. 2000 On the stability of the swept leading-edge boundary layer. PhD thesis, University of Washington, Seattle, WA.

Obrist, D. \& Schmid, P. J. $2003 a$ On the linear stability of swept attachment-line boundary layer flow. Part 1. Spectrum and asymptotic behaviour. J. Fluid Mech. 493, 1-29.

Oвrist, D. \& Schmid, P. J. $2003 b$ On the linear stability of swept attachment-line boundary layer flow. Part 2. Non-modal effects and receptivity. J. Fluid Mech. 493, 31-58.

Obrist, D. \& Schmid, P. J. 2009 Wave packet pseudomodes upstream of a swept cylinder. In Seventh IUTAM Symposium on Laminar-Turbulent Transition, Stockholm, Sweden.

SAlwen, H. \& Grosch, C. E. 1981 The continuous spectrum of the Orr-Sommerfeld equation. Part 2. Eigenfunction expansions. J. Fluid Mech. 104, 445-465. 
SPALART, P. R. 1988 Direct numerical study of leading-edge contamination. In Proceedings of the AGARD Symposium on Application of Direct and Large Eddy Simulation to Transition and Turbulence, $A G A R D$, CP-438, pp. 5-1-5-13.

THeofiLIs, V. 1998 On linear and nonlinear instability of the incompressible swept attachment-line boundary layer. J. Fluid Mech. 355, 193-227.

Trefethen, L. N. 2005 Wave packet pseudomodes of variable coefficient differential operators. Proc. R. Soc. A 461 (2062), 3099-3122.

Trefethen, L. N. \& Embree, M. 2005 Spectra and Pseudospectra: The Behaviour of Nonnormal Matrices and Operators. Princeton University Press.

Wilson, S. D. R. \& Gladwell, I. 1978 The stability of a two-dimensional stagnation flow to three-dimensional disturbances. J. Fluid Mech. 84, 517-527. 